\title{
Involutory Hopf group-coalgebras and flat bundles over 3-manifolds
}

\author{
by
}

Alexis Virelizier (Berkeley, CA)

\begin{abstract}
Given a group $\pi$, we use involutory Hopf $\pi$-coalgebras to define a scalar invariant of flat $\pi$-bundles over 3-manifolds. When $\pi=1$, this invariant equals the one for 3 -manifolds constructed by Kuperberg from involutory Hopf algebras. We give examples which show that this invariant is non-trivial.
\end{abstract}

\section{Contents}

Introduction

3. Invariants of flat bundles over 3-manifolds 258

4. Examples 265

References

\section{INTRODUCTION}

This paper is part of a program initiated by Turaev [8], called Homotopy quantum field theories (HQFT), whose purpose is the study of quantum invariants for maps. An $n$-dimensional HQFT with target a space $X$ consists in associating a vector space $V(g)$ to any map $g: N \rightarrow X$, where $N$ is a closed $(n-1)$-manifold, and a linear map $L(f): V\left(f_{\mid \partial_{-} M}\right) \rightarrow V\left(f_{\mid \partial_{+} M}\right)$ to any map $f: M \rightarrow X$, where $M$ is an $n$-cobordism with $\partial M=\partial_{+} M \cup\left(-\partial_{-} M\right)$. In particular, this assignment must only depend on the homotopy classes of the maps and must have the property that to compose two such cobordisms amounts to composing their associated linear maps. When $X$ is reduced to a single point, one recovers the notion of a topological quantum field theory, as described in [1].

Fix a group $\pi$. A HQFT with target an Eilenberg-Mac Lane space of type $K(\pi, 1)$ gives rise to invariants of flat $\pi$-bundles. The "quantum" versions of 3-manifold invariants can be generalized to this setting to get invariants

2000 Mathematics Subject Classification: 57M27, 16W30. 
of flat $\pi$-bundles over 3-manifolds (see [9] for Reshetikhin-Turaev's one and [11] for Hennings-Kauffman-Radford's one). The aim of this paper is to generalize the invariants of 3-manifolds constructed by Kuperberg [4] from involutory Hopf algebras to invariants of flat $\pi$-bundles over 3-manifolds.

The algebraic notion we use to replace that of a Hopf algebra is the notion of a Hopf $\pi$-coalgebra, introduced by Turaev in [9] (see also [12, 13]). Briefly speaking, a Hopf $\pi$-coalgebra is a family $H=\left\{H_{\alpha}\right\}_{\alpha \in \pi}$ of algebras (over a field $\mathbb{k}$ ) endowed with a comultiplication $\Delta=\left\{\Delta_{\alpha, \beta}: H_{\alpha \beta} \rightarrow H_{\alpha} \otimes H_{\beta}\right\}_{\alpha, \beta \in \pi}$, a counit $\varepsilon: H_{1} \rightarrow \mathbb{k}$, and an antipode $S=\left\{S_{\alpha}: H_{\alpha} \rightarrow H_{\alpha^{-1}}\right\}_{\alpha \in \pi}$ which satisfy some compatibility conditions. The case $\pi=1$ is the standard setting of Hopf algebras.

Fix a Hopf $\pi$-coalgebra $H=\left\{H_{\alpha}\right\}_{\alpha \in \pi}$ which is involutory, that is, such that its antipode satisfies $S_{\alpha^{-1}} S_{\alpha}=\operatorname{id}_{H_{\alpha}}$ for all $\alpha \in \pi$. Let $\xi=(p: \widetilde{M} \rightarrow M)$ be a flat $\pi$-bundle over a 3 -manifold $M$. Suppose that $\xi$ is pointed and denote its base point by $\widetilde{x} \in \widetilde{M}$. Let $x=p(\widetilde{x}) \in M$. Then there is a uniquely defined homomorphism $\pi_{1}(M, x) \rightarrow \pi$, called monodromy of $\xi$ at $\widetilde{x}$. We define a scalar invariant $K_{H}(\xi, \widetilde{x})$ of the pointed flat $\pi$-bundle $(\xi, \widetilde{x})$ as follows: we represent the base space $M$ of $\xi$ by a Heegaard diagram, we color this diagram with $\pi$ by using the monodromy of $\xi$ at $\widetilde{x}$, and we associate to this " $\pi$-colored" Heegaard diagram some structure constants of $H=\left\{H_{\alpha}\right\}_{\alpha \in \pi}$. The proof of this result consists in showing that the " $\pi$-colored" Reidemeister-Singer moves report the equivalence of pointed flat $\pi$-bundles over 3-manifolds, and in verifying the invariance of $K_{H}$ under these moves by using the properties of involutory Hopf $\pi$-coalgebras.

The invariant $K_{H}$ is non-trivial (we give examples of computation).

We study the dependence of $K_{H}(\xi, \widetilde{x})$ on the base point $\widetilde{x}$. In particular, we show that $K_{H}(\xi, \widetilde{x})$ only depends on the path-connected component of $\widetilde{x}$ and that $K_{H}(\xi, \widetilde{x})$ is independent of the choice of $\widetilde{x}$ when $\pi$ is abelian.

If $\pi=1$ and $M$ is a 3-manifold, then $K_{H}\left(\operatorname{id}_{M}: M \rightarrow M\right)$ coincides with the invariant of $M$ constructed by Kuperberg [4].

This paper is organized as follows. In Section 1, we review the properties of Hopf $\pi$-coalgebras. In Section 2, we construct an invariant of $\pi$-colored Heegaard diagrams. In Section 3, we show that this invariant yields an invariant of pointed flat $\pi$-bundles over 3-manifolds. Finally, we give examples in Section 4.

Acknowledgements. The author thanks Vladimir Turaev for useful suggestions and advice, and B. Enriquez and L. Vainerman for helpful conversations concerning the algebraic part.

Throughout this paper, $\pi$ is a group (with neutral element 1 ) and $\mathbb{k}$ is a field. All algebras are supposed to be over $\mathbb{k}$, associative, and unitary. 
The tensor product $\otimes=\otimes_{\mathbb{k}}$ is always assumed to be over $\mathbb{k}$. If $U$ and $V$ are $\mathbb{k}$-spaces, $\sigma_{U, V}: U \otimes V \rightarrow V \otimes U$ will denote the flip defined by $\sigma_{U, V}(u \otimes v)=v \otimes u$ for all $u \in U$ and $v \in V$.

\section{HOPF GROUP-COALGEBRAS}

In this section, we review definitions and properties concerning Hopf group-coalgebras. For a detailed treatment, we refer to [12].

1.1. Hopf $\pi$-coalgebras. Following [9], a Hopf $\pi$-coalgebra (over $\mathbb{k}$ ) is a family $H=\left\{H_{\alpha}\right\}_{\alpha \in \pi}$ of $\mathbb{k}$-algebras endowed with a family $\Delta=\left\{\Delta_{\alpha, \beta}: H_{\alpha \beta}\right.$ $\left.\rightarrow H_{\alpha} \otimes H_{\beta}\right\}_{\alpha, \beta \in \pi}$ of algebra homomorphisms (the comultiplication) and an algebra homomorphism $\varepsilon: H_{1} \rightarrow \mathbb{k}$ (the counit) such that, for all $\alpha, \beta, \gamma \in \pi$,

$$
\left(\Delta_{\alpha, \beta} \otimes \operatorname{id}_{H_{\gamma}}\right) \Delta_{\alpha \beta, \gamma}=\left(\operatorname{id}_{H_{\alpha}} \otimes \Delta_{\beta, \gamma}\right) \Delta_{\alpha, \beta \gamma}
$$

and

$$
\left(\operatorname{id}_{H_{\alpha}} \otimes \varepsilon\right) \Delta_{\alpha, 1}=\operatorname{id}_{H_{\alpha}}=\left(\varepsilon \otimes \operatorname{id}_{H_{\alpha}}\right) \Delta_{1, \alpha},
$$

and with a family $S=\left\{S_{\alpha}: H_{\alpha} \rightarrow H_{\alpha^{-1}}\right\}_{\alpha \in \pi}$ of $\mathbb{k}$-linear maps (the antipode) such that, for all $\alpha \in \pi$,

$$
m_{\alpha}\left(S_{\alpha^{-1}} \otimes \operatorname{id}_{H_{\alpha}}\right) \Delta_{\alpha^{-1}, \alpha}=\varepsilon 1_{\alpha}=m_{\alpha}\left(\operatorname{id}_{H_{\alpha}} \otimes S_{\alpha^{-1}}\right) \Delta_{\alpha, \alpha^{-1}},
$$

where $m_{\alpha}: H_{\alpha} \otimes H_{\alpha} \rightarrow H_{\alpha}$ and $1_{\alpha} \in H_{\alpha}$ denote the multiplication and unit element of $H_{\alpha}$.

When $\pi=1$, one recovers the usual notion of a Hopf algebra. In particular $H_{1}$ is a Hopf algebra.

Observe that the notion of a Hopf $\pi$-coalgebra is not self-dual, and if $H=\left\{H_{\alpha}\right\}_{\alpha \in \pi}$ is a Hopf $\pi$-coalgebra, then $\left\{\alpha \in \pi \mid H_{\alpha} \neq 0\right\}$ is a subgroup of $\pi$.

A Hopf $\pi$-coalgebra $H=\left\{H_{\alpha}\right\}_{\alpha \in \pi}$ is said to be of finite type if, for all $\alpha \in \pi, H_{\alpha}$ is finite-dimensional (over $\mathbb{k}$ ). Note that this does not mean that $\bigoplus_{\alpha \in \pi} H_{\alpha}$ is finite-dimensional (unless $H_{\alpha}=0$ for all but a finite number of $\alpha \in \pi)$.

The antipode of a Hopf $\pi$-coalgebra $H=\left\{H_{\alpha}\right\}_{\alpha \in \pi}$ is anti-multiplicative (the map $S_{\alpha}: H_{\alpha} \rightarrow H_{\alpha^{-1}}$ is an anti-homomorphism of algebras) and anticomultiplicative in the sense that $\Delta_{\beta^{-1}, \alpha^{-1}} S_{\alpha \beta}=\sigma_{H_{\alpha^{-1}}, H_{\beta^{-1}}}\left(S_{\alpha} \otimes S_{\beta}\right) \Delta_{\alpha, \beta}$ and $\varepsilon S_{1}=\varepsilon$ (see [12, Lemma 1.1]).

The antipode $S=\left\{S_{\alpha}\right\}_{\alpha \in \pi}$ of $H=\left\{H_{\alpha}\right\}_{\alpha \in \pi}$ is said to be bijective if each $S_{\alpha}$ is bijective. As for Hopf algebras, the antipode of a finite type Hopf $\pi$-coalgebra is always bijective (see [12, Corollary 3.7(a)]).

We extend the Sweedler notation for comultiplication to the setting of a Hopf $\pi$-coalgebra $H=\left\{H_{\alpha}\right\}_{\alpha \in \pi}$ as follows: for any $\alpha, \beta \in \pi$ and $h \in H_{\alpha \beta}$, we write $\Delta_{\alpha, \beta}(h)=\sum_{(h)} h_{(1, \alpha)} \otimes h_{(2, \beta)} \in H_{\alpha} \otimes H_{\beta}$, or briefly, if we leave 
the summation implicit, $\Delta_{\alpha, \beta}(h)=h_{(1, \alpha)} \otimes h_{(2, \beta)}$. The coassociativity of $\Delta$ gives that, for any $\alpha, \beta, \gamma \in \pi$ and $h \in H_{\alpha \beta \gamma}$,

$$
h_{(1, \alpha \beta)(1, \alpha)} \otimes h_{(1, \alpha \beta)(2, \beta)} \otimes h_{(2, \gamma)}=h_{(1, \alpha)} \otimes h_{(2, \beta \gamma)(1, \beta)} \otimes h_{(2, \beta \gamma)(2, \gamma)} .
$$

This element of $H_{\alpha} \otimes H_{\beta} \otimes H_{\gamma}$ is written as $h_{(1, \alpha)} \otimes h_{(2, \beta)} \otimes h_{(3, \gamma)}$. By iterating the procedure, we define inductively $h_{\left(1, \alpha_{1}\right)} \otimes \cdots \otimes h_{\left(n, \alpha_{n}\right)}$ for any $h \in H_{\alpha_{1} \cdots \alpha_{n}}$.

If $H=\left\{H_{\alpha}\right\}_{\alpha \in \pi}$ is a Hopf $\pi$-coalgebra with bijective antipode and $H_{\alpha}^{\mathrm{op}}$ denotes the opposite algebra to $H_{\alpha}$, then $H^{\mathrm{op}}=\left\{H_{\alpha}^{\mathrm{op}}\right\}_{\alpha \in \pi}$, endowed with the comultiplication and counit of $H$ and with the antipode $S^{\mathrm{op}}=\left\{S_{\alpha}^{\mathrm{op}}=\right.$ $\left.S_{\alpha^{-1}}^{-1}\right\}_{\alpha \in \pi}$, is a Hopf $\pi$-coalgebra, called opposite to $H$.

If $H=\left\{H_{\alpha}\right\}_{\alpha \in \pi}$ is a Hopf $\pi$-coalgebra with bijective antipode, set $H_{\alpha}^{\text {cop }}=H_{\alpha^{-1}}$ as an algebra, $\Delta_{\alpha, \beta}^{\text {cop }}=\sigma_{H_{\beta^{-1}}, H_{\alpha}-1} \Delta_{\beta^{-1}, \alpha^{-1}}, \varepsilon^{\text {cop }}=\varepsilon$, and $S_{\alpha}^{\text {cop }}=S_{\alpha}^{-1}$. Then $H^{\text {cop }}=\left\{H_{\alpha}^{\text {cop }}\right\}_{\alpha \in \pi}$ is a Hopf $\pi$-coalgebra, called coopposite to $H$.

1.2. The case of $\pi$ finite. Suppose $\pi$ is a finite group. Recall that the Hopf algebra $F(\pi)=k^{\pi}$ of functions on $\pi$ has a basis $\left(e_{\alpha}: \pi \rightarrow \mathbb{k}\right)_{\alpha \in \pi}$ defined by $e_{\alpha}(\beta)=\delta_{\alpha, \beta}$ where $\delta_{\alpha, \alpha}=1$ and $\delta_{\alpha, \beta}=0$ if $\alpha \neq \beta$. The structure maps of $F(\pi)$ are given by $e_{\alpha} e_{\beta}=\delta_{\alpha, \beta} e_{\alpha}, 1_{F(\pi)}=\sum_{\alpha \in \pi} e_{\alpha}, \Delta\left(e_{\alpha}\right)=\sum_{\beta \gamma=\alpha} e_{\beta} \otimes e_{\gamma}$, $\varepsilon\left(e_{\alpha}\right)=\delta_{\alpha, 1}$, and $S\left(e_{\alpha}\right)=e_{\alpha^{-1}}$.

By a central prolongation of $F(\pi)$ we shall mean a Hopf algebra $A$ endowed with a morphism of Hopf algebras $F(\pi) \rightarrow A$ which sends $F(\pi)$ into the center of $A$. The morphism $F(\pi) \rightarrow A$ is called the central map of $A$.

Since $\pi$ is finite, any Hopf $\pi$-coalgebra $H=\left\{H_{\alpha}\right\}_{\alpha \in \pi}$ gives rise to a Hopf algebra $\widetilde{H}=\bigoplus_{\alpha \in \pi} H_{\alpha}$ with structure maps given by $\left.\widetilde{\Delta}\right|_{H_{\alpha}}=\sum_{\beta \gamma=\alpha} \Delta_{\beta, \gamma}$, $\left.\widetilde{\varepsilon}\right|_{H_{\alpha}}=\delta_{\alpha, 1} \varepsilon,\left.\widetilde{m}\right|_{H_{\alpha} \otimes H_{\beta}}=\delta_{\alpha, \beta} m_{\alpha}, \widetilde{1}=\sum_{\alpha \in \pi} 1_{\alpha}$, and $\widetilde{S}=\sum_{\alpha \in \pi} S_{\alpha}$.

The $\mathbb{k}$-linear map $F(\pi) \rightarrow \widetilde{H}$ defined by $e_{\alpha} \mapsto 1_{\alpha}$ clearly gives rise to a morphism of Hopf algebras which sends $F(\pi)$ into the center of $\widetilde{H}$. Hence $\widetilde{H}$ is a central prolongation of $F(\pi)$.

As noticed by Enriquez [2], the correspondence which assigns to every Hopf $\pi$-coalgebra $H=\left\{H_{\alpha}\right\}_{\alpha \in \pi}$ the central prolongation $\widetilde{H}$ of $F(\pi)$ is one-to-one. Indeed, let $(A, m, 1, \Delta, \varepsilon, S)$ be a central prolongation of $F(\pi)$. Denote by $1_{\alpha} \in A$ the image of $e_{\alpha} \in F(\pi)$ under the central map $F(\pi) \rightarrow A$ of $A$. Set $H_{\alpha}=A 1_{\alpha}$ for any $\alpha \in \pi$. Since $F(\pi) \rightarrow A$ is a morphism of Hopf algebras and each $1_{\alpha} \in A$ is central, the family $\left\{H_{\alpha}\right\}_{\alpha \in \pi}$ is a Hopf $\pi$-coalgebra with structure maps given by $m_{\alpha}=\left.1_{\alpha} \cdot m\right|_{H_{\alpha} \otimes H_{\alpha}}, \Delta_{\alpha, \beta}=\left.\left(1_{\alpha} \otimes 1_{\beta}\right) \cdot \Delta\right|_{H_{\alpha \beta}}$, $\varepsilon=\left.\varepsilon\right|_{H_{1}}$, and $S_{\alpha}=\left.1_{\alpha^{-1}} \cdot S\right|_{H_{\alpha}}$. Furthermore, $\widetilde{H}=A$ as a central prolongation of $F(\pi)$, where $\widetilde{H}=\bigoplus_{\alpha \in \pi} H_{\alpha}$ is the central prolongation of $F(\pi)$ associated to $\left\{H_{\alpha}\right\}_{\alpha \in \pi}$ as above. 
1.3. $\pi$-integrals. Recall that a left (resp. right) integral for a Hopf algebra $(A, \Delta, \varepsilon, S)$ is an element $\Lambda \in A$ such that $x \Lambda=\varepsilon(x) \Lambda$ (resp. $\Lambda x=$ $\varepsilon(x) \Lambda)$ for all $x \in A$. A left (resp. right) integral for the dual Hopf algebra $A^{*}$ is a $\mathbb{k}$-linear form $\lambda \in A^{*}$ satisfying $\left(\mathrm{id}_{A} \otimes \lambda\right) \Delta(x)=\lambda(x) 1_{A}$ (resp. $\left.\left(\lambda \otimes \operatorname{id}_{A}\right) \Delta(x)=\lambda(x) 1_{A}\right)$ for all $x \in A$.

By a left (resp. right) $\pi$-integral for a Hopf $\pi$-coalgebra $H=\left\{H_{\alpha}\right\}_{\alpha \in \pi}$, we shall mean a family $\lambda=\left(\lambda_{\alpha}\right)_{\alpha \in \pi} \in \prod_{\alpha \in \pi} H_{\alpha}^{*}$ of $\mathbb{k}$-linear forms such that

$$
\left(\operatorname{id}_{H_{\alpha}} \otimes \lambda_{\beta}\right) \Delta_{\alpha, \beta}(x)=\lambda_{\alpha \beta}(x) 1_{\alpha}\left(\operatorname{resp} .\left(\lambda_{\alpha} \otimes \operatorname{id}_{H_{\beta}}\right) \Delta_{\alpha, \beta}(x)=\lambda_{\alpha \beta}(x) 1_{\beta}\right)
$$

for all $\alpha, \beta \in \pi$ and $x \in H_{\alpha \beta}$.

Note that $\lambda_{1}$ is a usual left (resp. right) integral for the Hopf algebra $H_{1}^{*}$.

A $\pi$-integral $\lambda=\left(\lambda_{\alpha}\right)_{\alpha \in \pi}$ is said to be non-zero if $\lambda_{\beta} \neq 0$ for some $\beta \in \pi$. Note that a non-zero $\pi$-integral $\lambda=\left(\lambda_{\alpha}\right)_{\alpha \in \pi}$ has $\lambda_{\alpha} \neq 0$ for all $\alpha \in \pi$ such that $H_{\alpha} \neq 0$. In particular $\lambda_{1} \neq 0$.

It is known that the space of left (resp. right) integrals for a finitedimensional Hopf algebra is one-dimensional. In the setting of Hopf $\pi$-coalgebras, the space of left (resp. right) $\pi$-integrals for a finite type Hopf $\pi$-coalgebra is one-dimensional (even when $\pi$ is infinite; see [12, Theorem 3.6]).

1.4. Semisimplicity. A Hopf $\pi$-coalgebra $H=\left\{H_{\alpha}\right\}_{\alpha \in \pi}$ is said to be semisimple if each algebra $H_{\alpha}$ is semisimple. Note that a necessary condition for $H$ to be semisimple is that $H_{1}$ is finite-dimensional (since an infinite-dimensional Hopf algebra over a field is never semisimple, see [7, Corollary 2.7]). If $H$ is of finite type, then $H$ is semisimple if and only if $H_{1}$ is semisimple (see [12, Lemma 5.1]).

1.5. Cosemisimplicity. The notion of a comodule over a coalgebra may be extended to the setting of Hopf $\pi$-coalgebras. Namely, a right $\pi$-comodule over a Hopf $\pi$-coalgebra $H=\left\{H_{\alpha}\right\}_{\alpha \in \pi}$ is a family $M=\left\{M_{\alpha}\right\}_{\alpha \in \pi}$ of $\mathbb{k}$-spaces endowed with a family $\varrho=\left\{\varrho_{\alpha, \beta}: M_{\alpha \beta} \rightarrow M_{\alpha} \otimes H_{\beta}\right\}_{\alpha, \beta \in \pi}$ of $\mathbb{k}$-linear maps such that $\left(\varrho_{\alpha, \beta} \otimes \operatorname{id}_{H_{\gamma}}\right) \varrho_{\alpha \beta, \gamma}=\left(\operatorname{id}_{M_{\alpha}} \otimes \Delta_{\beta, \gamma}\right) \varrho_{\alpha, \beta \gamma}$ and $\left(\operatorname{id}_{M_{\alpha}} \otimes \varepsilon\right) \varrho_{\alpha, 1}=\operatorname{id}_{M_{\alpha}}$ for all $\alpha, \beta, \gamma \in \pi$. A $\pi$-subcomodule of $M$ is a family $N=\left\{N_{\alpha}\right\}_{\alpha \in \pi}$, where $N_{\alpha}$ is a $\mathbb{k}$-subspace of $M_{\alpha}$, such that $\varrho_{\alpha, \beta}\left(N_{\alpha \beta}\right) \subset N_{\alpha} \otimes H_{\beta}$ for all $\alpha, \beta \in \pi$. The notion of sum and direct sum of a family of $\pi$-subcomodules of a right $\pi$-comodule may be defined in the obvious way.

A right $\pi$-comodule $M=\left\{M_{\alpha}\right\}_{\alpha \in \pi}$ is said to be simple if it is non-zero (i.e., $M_{\alpha} \neq 0$ for some $\alpha \in \pi$ ) and if it has no $\pi$-subcomodules other than $0=\{0\}_{\alpha \in \pi}$ and itself. A right $\pi$-comodule which is a direct sum of a family of simple $\pi$-subcomodules is said to be cosemisimple. A Hopf $\pi$-coalgebra is called cosemisimple if it is cosemisimple as a right $\pi$-comodule over itself (with comultiplication as structure maps). 
There exists (see [12, Theorem 5.4]) a Hopf $\pi$-coalgebra version of the dual Maschke theorem: a Hopf $\pi$-coalgebra $H=\left\{H_{\alpha}\right\}_{\alpha \in \pi}$ is cosemisimple if and only if there exists a right $\pi$-integral $\lambda=\left(\lambda_{\alpha}\right)_{\alpha \in \pi}$ for $H$ such that $\lambda_{\alpha}\left(1_{\alpha}\right)=1$ for all $\alpha \in \pi$ with $H_{\alpha} \neq 0$. In particular, if $H$ is of finite type, then $H$ is cosemisimple if and only if the Hopf algebra $H_{1}$ is cosemisimple.

1.6. Crossed Hopf $\pi$-coalgebras. The notion of a crossing for a Hopf $\pi$-coalgebra is needed to define the quasitriangularity of a Hopf $\pi$-coalgebra (see $[9,12]$ ). A Hopf $\pi$-coalgebra $H=\left\{H_{\alpha}\right\}_{\alpha \in \pi}$ is said to be crossed if it is endowed with a family $\varphi=\left\{\varphi_{\beta}: H_{\alpha} \rightarrow H_{\beta \alpha \beta^{-1}}\right\}_{\alpha, \beta \in \pi}$ of algebra isomorphisms (the crossing) such that each $\varphi_{\beta}$ preserves the comultiplication and the counit, i.e., $\left(\varphi_{\beta} \otimes \varphi_{\beta}\right) \Delta_{\alpha, \gamma}=\Delta_{\beta \alpha \beta^{-1}, \beta \gamma \beta^{-1}} \varphi_{\beta}$ and $\varepsilon \varphi_{\beta}=\varepsilon$ for all $\alpha, \beta, \gamma \in \pi$, and $\varphi$ is multiplicative in the sense that $\varphi_{\beta \beta^{\prime}}=\varphi_{\beta} \varphi_{\beta^{\prime}}$ for all $\beta, \beta^{\prime} \in \pi$.

One easily verifies that a crossing preserves the antipode, that is, $\varphi_{\beta} S_{\alpha}=$ $S_{\beta \alpha \beta^{-1}} \varphi_{\beta}$ for all $\alpha, \beta \in \pi$.

A particular class of crossed Hopf $\pi$-coalgebras is that of Hopf $\pi$-coalgebras with $\pi$ abelian: if $\pi$ is an abelian group, then a Hopf $\pi$-coalgebra $H=\left\{H_{\alpha}\right\}_{\alpha \in \pi}$ is always crossed (e.g., by taking $\left.\varphi_{\beta}\right|_{H_{\alpha}}=\mathrm{id}_{H_{\alpha}}$ ).

When $\pi$ is a finite group, the notion of a crossing can be described by using the language of central prolongations of $F(\pi)$ (see Section 1.2). More precisely, a central prolongation $A$ of $F(\pi)$ is crossed if it is endowed with a group homomorphism $\varphi: \pi \rightarrow \operatorname{Aut}_{\text {Hopf }}(A)$ such that $\varphi_{\beta}\left(1_{\alpha}\right)=1_{\beta \alpha \beta^{-1}}$ for all $\alpha, \beta \in \pi$, where $\operatorname{Aut}_{\text {Hopf }}(A)$ is the group of Hopf automorphisms of the Hopf algebra $A$ and $1_{\alpha}$ denotes the image of $e_{\alpha} \in F(\pi)$ under the central $\operatorname{map} F(\pi) \rightarrow A$.

For examples of crossed Hopf $\pi$-coalgebras, we refer to [13].

1.7. Involutory Hopf $\pi$-coalgebras. In this section we give some results concerning involutory Hopf $\pi$-coalgebras which are used for topological purposes in Sections 2 and 3.

A Hopf $\pi$-coalgebra $H=\left\{H_{\alpha}\right\}_{\alpha \in \pi}$ is said to be involutory if its antipode $S=\left\{S_{\alpha}\right\}_{\alpha \in \pi}$ is such that $S_{\alpha^{-1}} S_{\alpha}=\operatorname{id}_{H_{\alpha}}$ for all $\alpha \in \pi$.

If $A$ is an algebra and $a \in A$, then $r(a) \in \operatorname{End}_{\mathbb{k}}(A)$ will denote the right multiplication by $a$ defined by $r(a)(x)=x a$ for any $x \in A$. Moreover, $\operatorname{Tr}$ will denote the usual trace of $\mathbb{k}$-linear endomorphisms of a $\mathbb{k}$-space.

Lemma 1. Let $H=\left\{H_{\alpha}\right\}_{\alpha \in \pi}$ be a finite type Hopf $\pi$-coalgebra with antipode $S=\left\{S_{\alpha}\right\}_{\alpha \in \pi}$. Let $\lambda=\left(\lambda_{\alpha}\right)_{\alpha \in \pi}$ be a right $\pi$-integral for $H$ and $\Lambda$ be a left integral for $H_{1}$ such that $\lambda_{1}(\Lambda)=1$. Let $\alpha \in \pi$. Then

(a) $\operatorname{Tr}(f)=\lambda_{\alpha}\left(S_{\alpha^{-1}}\left(\Lambda_{\left(2, \alpha^{-1}\right)}\right) f\left(\Lambda_{(1, \alpha)}\right)\right)$ for all $f \in \operatorname{End}_{\mathbb{k}}\left(H_{\alpha}\right)$;

(b) $\operatorname{Tr}\left(r(a) \circ S_{\alpha^{-1}} S_{\alpha}\right)=\varepsilon(\Lambda) \lambda_{\alpha}(a)$ for all $a \in H_{\alpha}$; 
(c) If $H_{\alpha} \neq 0$, then $\operatorname{Tr}\left(S_{\alpha^{-1}} S_{\alpha}\right) \neq 0$ if and only if $H$ is semisimple and cosemisimple;

(d) If $H_{\alpha} \neq 0$, then $\operatorname{Tr}\left(S_{\alpha^{-1}} S_{\alpha}\right)=\operatorname{Tr}\left(S_{1}^{2}\right)$.

Proof. To show (a), identify $H_{\alpha}^{*} \otimes H_{\alpha}$ and $\operatorname{End}_{\mathbb{k}}\left(H_{\alpha}\right)$ by $(p \otimes a)(x)=$ $p(x) a$ for all $p \in H_{\alpha}$ and $a, x \in H_{\alpha}$. Under this identification, $\operatorname{Tr}(p \otimes a)=$ $p(a)$. Let $f \in \operatorname{End}_{\mathbb{k}}\left(H_{\alpha}\right)$. We may assume that $f=p \otimes a$ for some $p \in$ $H_{\alpha}^{*}$ and $a \in H_{\alpha}$. By [12, Corollary 3.7(b)], there exists $b \in H_{\alpha}$ such that $p(x)=\lambda_{\alpha}(b x)$ for all $x \in H_{\alpha}$. Now $S_{\alpha^{-1}}^{-1}(b)=\lambda_{\alpha}\left(b \Lambda_{(1, \alpha)}\right) \Lambda_{\left(2, \alpha^{-1}\right)}$ by $[12$, Lemma 4.3(b)] and so

Therefore

$$
b=\lambda_{\alpha}\left(b \Lambda_{(1, \alpha)}\right) S_{\alpha^{-1}}\left(\Lambda_{\left(2, \alpha^{-1}\right)}\right)=p\left(\Lambda_{(1, \alpha)}\right) S_{\alpha^{-1}}\left(\Lambda_{\left(2, \alpha^{-1}\right)}\right) .
$$

$$
\begin{aligned}
\operatorname{Tr}(f) & =p(a)=\lambda_{\alpha}(b a)=\lambda_{\alpha}\left(S_{\alpha^{-1}}\left(\Lambda_{\left(2, \alpha^{-1}\right)}\right) p\left(\Lambda_{(1, \alpha)}\right) a\right) \\
& =\lambda_{\alpha}\left(S_{\alpha^{-1}}\left(\Lambda_{\left(2, \alpha^{-1}\right)}\right) f\left(\Lambda_{(1, \alpha)}\right)\right) .
\end{aligned}
$$

Let us show (b). Let $a \in H_{\alpha}$. Then

$$
\begin{aligned}
\operatorname{Tr}\left(r(a) \circ S_{\alpha^{-1}} S_{\alpha}\right) & =\lambda_{\alpha}\left(S_{\alpha^{-1}}\left(\Lambda_{\left(2_{\alpha^{-1}}\right)}\right) S_{\alpha^{-1}} S_{\alpha}\left(\Lambda_{(1, \alpha)}\right) a\right) \quad \text { by (a) } \\
& =\lambda_{\alpha}\left(S_{\alpha^{-1}}\left(S_{\alpha}\left(\Lambda_{(1, \alpha)}\right) \Lambda_{\left(2_{\alpha^{-1}}\right)}\right) a\right) \\
& =\lambda_{\alpha}\left(S_{\alpha^{-1}}\left(\varepsilon(\Lambda) 1_{\alpha^{-1}}\right) a\right) \quad \text { by }(3) \\
& =\varepsilon(\Lambda) \lambda_{\alpha}(a) .
\end{aligned}
$$

To show (c), suppose $H_{\alpha} \neq 0$. Since $\operatorname{Tr}\left(S_{\alpha^{-1}} S_{\alpha}\right)=\varepsilon(\Lambda) \lambda_{\alpha}\left(1_{\alpha}\right)$ (by (b)), one easily concludes using the facts that $H$ is semisimple if and only if $\varepsilon(\Lambda) \neq 0$ (by [12, Lemma 5.1] and [6, Theorem 5.1.8]), and $H$ is cosemisimple if and only if $\lambda_{\alpha}\left(1_{\alpha}\right) \neq 0$ (by [12, Theorem 5.4] since $H_{\alpha} \neq 0$ ).

Let us show (d). By using (4), we have

$$
\lambda_{1}\left(1_{1}\right) 1_{\alpha}=\left(\lambda_{1} \otimes \operatorname{id}_{H_{\alpha}}\right) \Delta_{1, \alpha}\left(1_{\alpha}\right)=\lambda_{\alpha}\left(1_{\alpha}\right) 1_{\alpha},
$$

and so $\lambda_{\alpha}\left(1_{\alpha}\right)=\lambda_{1}\left(1_{1}\right)$ since $1_{\alpha} \neq 0$ (because $\left.H_{\alpha} \neq 0\right)$. Hence, by (b), we conclude that $\operatorname{Tr}\left(S_{\alpha^{-1}} S_{\alpha}\right)=\varepsilon(\Lambda) \lambda_{\alpha}\left(1_{\alpha}\right)=\varepsilon(\Lambda) \lambda_{1}\left(1_{1}\right)=\operatorname{Tr}\left(S_{1}^{2}\right)$.

Lemma 2. Let $H=\left\{H_{\alpha}\right\}_{\alpha \in \pi}$ be a finite type involutory Hopf $\pi$-coalgebra over a field of characteristic $p$. Let $\alpha \in \pi$ with $H_{\alpha} \neq 0$. If $p=0$ or $p>$ $\left|\operatorname{dim} H_{\alpha}-\operatorname{dim} H_{1}\right|$, then $\operatorname{dim} H_{\alpha}=\operatorname{dim} H_{1}$.

Proof. By Lemma $1(\mathrm{~d})$, we have $\operatorname{Tr}\left(S_{\alpha^{-1}} S_{\alpha}\right)=\operatorname{Tr}\left(S_{1}^{2}\right)$, that is, since $H$ is involutory, $\left(\operatorname{dim} H_{\alpha}\right) 1_{\mathbb{k}}=\left(\operatorname{dim} H_{1}\right) 1_{\mathbb{k}}$. One easily concludes by using the hypothesis on the characteristic of the field $\mathbb{k}$.

Lemma 3. Let $H=\left\{H_{\alpha}\right\}_{\alpha \in \pi}$ be a finite type involutory Hopf $\pi$-coalgebra. Suppose that $\operatorname{dim} H_{1} \neq 0$ in the ground field $\mathbb{k}$ of $H$. Then $H$ is semisimple and cosemisimple.

Proof. This is a consequence of Lemma $1(\mathrm{c})$ since $\operatorname{Tr}\left(S_{1}^{2}\right)=\operatorname{Tr}\left(\mathrm{id}_{H_{1}}\right)=$ $\operatorname{dim} H_{1} \neq 0$. 
1.8. Diagrammatic formalism of Hopf group-coalgebras. The structure maps of a Hopf $\pi$-coalgebra $H=\left\{H_{\alpha}\right\}_{\alpha \in \pi}$ can be represented symbolically as in [4]. The multiplications $m_{\alpha}: H_{\alpha} \otimes H_{\alpha} \rightarrow H_{\alpha}$, the unit elements $1_{\alpha}$, the comultiplication $\Delta_{\alpha, \beta}: H_{\alpha \beta} \rightarrow H_{\alpha} \otimes H_{\beta}$, the counit $\varepsilon: H_{1} \rightarrow \mathbb{k}$, and the antipode $S_{\alpha}: H_{\alpha} \rightarrow H_{\alpha^{-1}}$ are represented as in Figure 1. The inputs (incoming arrows) for the product symbols are read counterclockwise and the outputs arrows (outgoing arrows) for the comultiplication symbols are read clockwise.

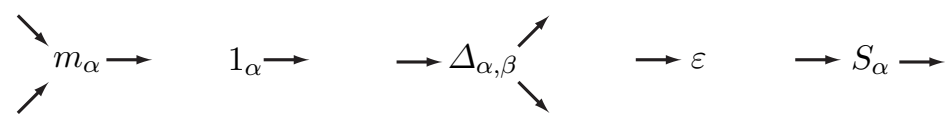

Fig. 1. The structure maps

In light of the associativity and coassociativity axioms (see Section 1.1), we adopt the abbreviations of Figure 2.

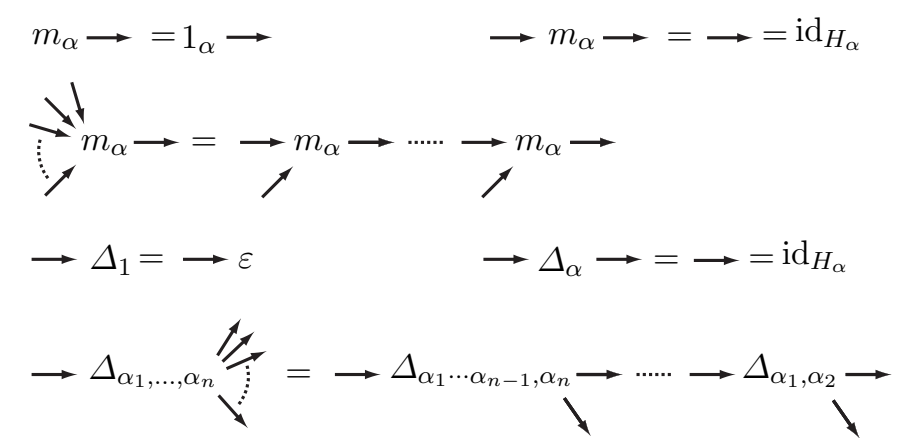

Fig. 2. Diagrammatic abbreviations

The combinatorics of the diagrams involving such symbolical representations of structure maps may be thought of as (sum of) products of structure constants. For example, if $\left(e_{i}\right)_{i}$ is a basis of $H_{1}$ and $\delta_{i}^{j, k} \in \mathbb{k}$ are the structure constants of $\Delta_{1,1}$ defined by $\Delta_{1,1}\left(e_{i}\right)=\sum_{j, k} \delta_{i}^{j, k} e_{j} \otimes e_{k}$, then the element $C \in H_{1}$ represented in Figure 3(a) is given by $C=\sum_{i, k} \delta_{i}^{i, k} e_{k}$.

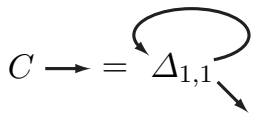

(a) $C \in H_{1}$

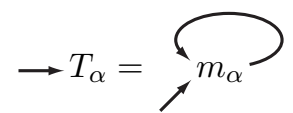

(b) $T_{\alpha}: H_{\alpha} \rightarrow \mathbb{k}$

Fig. 3 
Similarly, if $\left(e_{i}\right)_{i}$ is a basis of $H_{\alpha}$ and $\mu_{i, j}^{k} \in \mathbb{k}$ are the structure constants of $m_{\alpha}$ defined by $m_{\alpha}\left(e_{i} \otimes e_{j}\right)=\sum_{k} \mu_{i, j}^{k} e_{k}$, then the $\mathbb{k}$-linear form $T_{\alpha}: H_{\alpha} \rightarrow \mathbb{k}$ represented in Figure 3(b) is given by $T_{\alpha}\left(e_{i}\right)=\sum_{k} \mu_{k, i}^{k}$. Note that $T_{\alpha}(x)=\operatorname{Tr}(r(x))$ for any $x \in H_{\alpha}$, where $r(x) \in \operatorname{End}_{\mathbb{k}}\left(H_{\alpha}\right)$ denotes the right multiplication by $x$ and $\mathrm{Tr}$ is the usual trace of $\mathbb{k}$-linear endomorphisms.

Until the end of this section, $H=\left\{H_{\alpha}\right\}_{\alpha \in \pi}$ will denote a finite type involutory Hopf $\pi$-coalgebra with $\operatorname{dim} H_{1} \neq 0$ in the ground field $\mathbb{k}$ of $H$.

Lemma 4. $T=\left(T_{\alpha}\right)_{\alpha \in \pi}$ is a non-zero two-sided $\pi$-integral for $H$ and $C$ is a non-zero two-sided integral for $H_{1}$ which satisfy $T_{1}\left(1_{1}\right)=\varepsilon(C)=$ $T_{1}(C)=\operatorname{dim} H_{1}$. Moreover $S_{1}(C)=C$ and $T_{\alpha^{-1}} \circ S_{\alpha}=T_{\alpha}$ for all $\alpha \in \pi$.

Proof. Recall that $H$ is semisimple and cosemisimple (by Lemma 3). Therefore, by [12, Theorem 5.4 and Corollary 5.7], there exists a two-sided $\pi$-integral $\lambda=\left(\lambda_{\alpha}\right)_{\alpha \in \pi}$ for $H$ such that $\lambda_{\alpha}\left(1_{\alpha}\right)=1$ for all $\alpha \in \pi$ with $H_{\alpha} \neq 0$. Let $\Lambda$ be a left integral for $H_{1}$ such that $\lambda_{1}(\Lambda)=1$. By Lemma $1(\mathrm{~b})$, we have $T_{\alpha}(x)=\operatorname{Tr}(r(x))=\varepsilon(\Lambda) \lambda_{\alpha}(x)$ for any $x \in H_{\alpha}$. Therefore $T=$ $\left(T_{\alpha}\right)_{\alpha \in \pi}$ is a multiple of $\lambda=\left(\lambda_{\alpha}\right)_{\alpha \in \pi}$ and so is a two-sided $\pi$-integral for $H$, which is non-zero since $\varepsilon(\Lambda) \neq 0$ (because $H_{1}$ is semisimple, see [6, Theorem 5.1.8]). Likewise $C=\lambda_{1}\left(1_{1}\right) \Lambda=\Lambda$ (by Lemma 1(b) applied to the Hopf algebra $H_{1}^{*}$ ) and so $C$ is a non-zero left integral for $H_{1}$. Moreover $C$ is a right integral for $H_{1}$ (since $H_{1}$ is semisimple and so its integrals are two-sided).

Since $\lambda_{1}\left(1_{1}\right)=\lambda_{1}(\Lambda)=1$ and by Lemma $1(\mathrm{~b})$, we have $T_{1}(C)=T_{1}\left(1_{1}\right)=$ $\varepsilon(C)=\varepsilon(\Lambda)=\operatorname{Tr}\left(\operatorname{id}_{H_{1}}\right)=\operatorname{dim} H_{1}$.

Since $H$ is cosemisimple, [12, Theorem 4.2(c) and Corollary 5.7] give that $T_{\alpha^{-1}} \circ S_{\alpha}=T_{\alpha}$ for all $\alpha \in \pi$. Finally, $S_{1}(C)$ is a left integral for $H_{1}$ and so there exists $k \in \mathbb{k}$ such that $S_{1}(C)=k C$. Now $k=1$ since $C=\Lambda$, $\lambda_{1}(\Lambda)=1$, and $\lambda_{1} \circ S_{1}=\lambda_{1}$. Hence $S_{1}(C)=C$.

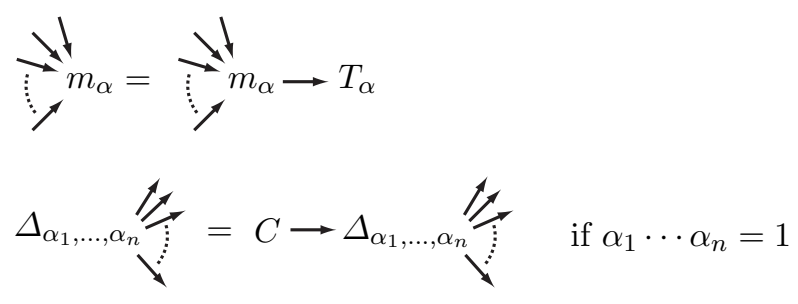

Fig. 4

Lemma 5. The two tensors represented by the diagrams of Figure 4 are cyclically symmetric. 
Proof. Let $\alpha \in \pi$. Since $\left(T_{\beta}\right)_{\beta \in \pi}$ is a right $\pi$-integral for $H$ (by Lemma 4 ) and the Hopf algebra $H_{1}$ is semisimple and so unimodular, [12, Theorem 4.2(a)] gives that, for all $x, y \in H_{\alpha}$,

$$
T_{\alpha}(x y)=T_{\alpha}\left(S_{\alpha^{-1}} S_{\alpha}(y) x\right)=T_{\alpha}(y x) .
$$

Hence $T_{\alpha}\left(x_{1} x_{2} \cdots x_{n}\right)=T_{\alpha}\left(x_{2} \cdots x_{n} x_{1}\right)$ for all $x_{1}, \ldots, x_{n} \in H_{\alpha}$.

Since $H$ is cosemisimple and $C$ is a left integral for $H_{1}$, by using [12, Corollaries 4.4 and 5.7] we find that, for all $\alpha \in \pi$,

$$
C_{(1, \alpha)} \otimes C_{\left(2, \alpha^{-1}\right)}=S_{\alpha^{-1}} S_{\alpha}\left(C_{(2, \alpha)}\right) 1_{\alpha} \otimes C_{\left(1, \alpha^{-1}\right)}=C_{(2, \alpha)} \otimes C_{\left(1, \alpha^{-1}\right)} .
$$

Therefore, for all $\alpha_{1}, \ldots, \alpha_{n} \in \pi$ such that $\alpha_{1} \cdots \alpha_{n}=1$, we obtain

$$
\begin{aligned}
C_{\left(1, \alpha_{1}\right)} \otimes \cdots \otimes & C_{\left(n-1, \alpha_{n-1}\right)} \otimes C_{\left(n, \alpha_{n}\right)} \\
& =\left(C_{\left(1, \alpha_{n}^{-1}\right)}\right)_{\left(1, \alpha_{1}\right)} \otimes \cdots \otimes\left(C_{\left(1, \alpha_{n}^{-1}\right)}\right)_{\left(n-1, \alpha_{n-1}\right)} \otimes C_{\left(2, \alpha_{n}\right)} \\
& =\left(C_{\left(2, \alpha_{n}^{-1}\right)}\right)_{\left(1, \alpha_{1}\right)} \otimes \cdots \otimes\left(C_{\left(2, \alpha_{n}^{-1}\right)}\right)_{\left(n-1, \alpha_{n-1}\right)} \otimes C_{\left(1, \alpha_{n}\right)} \\
& =C_{\left(2, \alpha_{1}\right)} \otimes \cdots \otimes C_{\left(n, \alpha_{n-1}\right)} \otimes C_{\left(1, \alpha_{n}\right)} .
\end{aligned}
$$

Hence the two tensors of Figure 4 are cyclically symmetric.

\section{INVARIANTS OF COLORED HEEGAARD DIAGRAMS}

In this section, we define $\pi$-colored Heegaard diagrams and their equivalence. Then, starting from an involutory Hopf $\pi$-coalgebra, we construct an equivalence invariant of $\pi$-colored Heegaard diagrams.

2.1. Colored Heegaard diagrams. By a Heegaard diagram, we shall mean a triple $D=(S, u, l)$ where $S$ is a closed, connected, and oriented surface of genus $g \geq 1$ and $u=\left\{u_{1}, \ldots, u_{g}\right\}$ and $l=\left\{l_{1}, \ldots, l_{g}\right\}$ are two systems of pairwise disjoint closed curves on $S$ such that the complement to $\bigcup_{k} u_{k}$ (resp. $\bigcup_{i} l_{i}$ ) is connected. Note that if a sphere with $g$ handles is cut along $g$ disjoint circles that do not split it, then a sphere from which $2 g$ disks have been deleted is obtained.

The circles $u_{k}$ (resp. $l_{i}$ ) are called the upper (resp. lower) circles of the diagram. By general position we can (and we always do) assume that $u$ and $l$ are transverse. Note that $u \cap l$ is then a finite set. The Heegaard diagram $D$ is said to be oriented if all its lower and upper circles are oriented.

Let $D=(S, u, l)$ be an oriented Heegaard diagram. Denote by $g$ the genus of $S$. Fix an alphabet $X=\left\{x_{1}, \ldots, x_{g}\right\}$ of $g$ letters. For any $1 \leq i \leq g$, traveling along the lower circle $l_{i}$ gives a word $w_{i}\left(x_{1}, \ldots, x_{g}\right)$ as follows:

- Start with the empty word $w_{i}=\emptyset$;

- Make a round trip along $l_{i}$ following its orientation. Each time $l_{i}$ encounters an upper circle $u_{k}$ at some crossing $c \in l_{i} \cap u_{k}$ (for some 
$1 \leq k \leq g)$, replace $w_{i}$ by $w_{i} x_{k}^{\nu}$ where

$$
\nu= \begin{cases}+1 & \text { if }\left(d_{c} l_{i}, d_{c} u_{k}\right) \text { is an oriented basis for } T_{c} S, \\ -1 & \text { otherwise }\end{cases}
$$

- After a complete turn along $l_{i}$, one gets $w_{j}$.

Note that the word $w_{i}$ is well defined up to conjugacy by some word in the letters $x_{1}, \ldots, x_{g}$ (this is due to the indeterminacy in the choice of the starting point on $l_{i}$ ).

We say that the Heegaard diagram $D$ is $\pi$-colored if each upper circle $u_{k}$ is provided with an element $\alpha_{k} \in \pi$ such that $w_{i}\left(\alpha_{1}, \ldots, \alpha_{g}\right)=1 \in \pi$ for all $1 \leq i \leq g$. The system $\alpha=\left(\alpha_{1}, \ldots, \alpha_{g}\right)$ is called the color of $D$.

Two $\pi$-colored Heegaard diagrams are said to be equivalent if one can be obtained from the other by a finite sequence of the following moves (or their inverses):

TYPE I (homeomorphism of the surface): By using an orientation-preserving homeomorphism of a (closed, connected, and oriented) surface $S$ to a (closed, connected, and oriented) surface $S^{\prime}$, the upper (resp. lower) circles on $S$ are carried to the upper (resp. lower) circles on $S^{\prime}$. The colors of the upper circles remain unchanged.

TYPE II (orientation reversal): The orientation of an upper or lower circle is changed to its opposite. For an upper circle $u_{i}$, its color $\alpha_{i}$ is changed to its inverse $\alpha_{i}^{-1}$.

TYPE III (isotopy of the diagram): We isotope the lower circles of the diagram relative to the upper circles. If this isotopy is in general position, it reduces to a sequence of two-point moves shown in Figure 5. The colors of the upper circles remain unchanged.

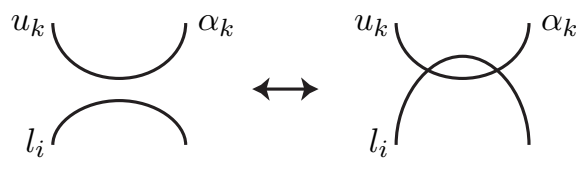

Fig. 5. Two-point move

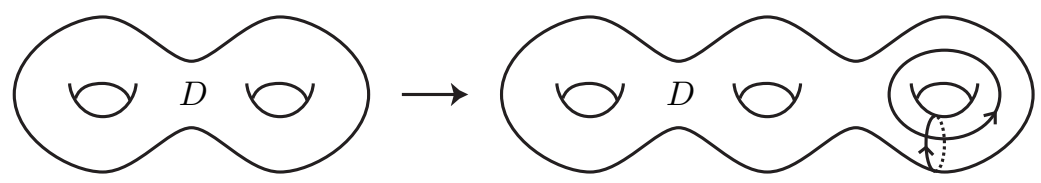

Fig. 6. Stabilization 
TyPE IV (stabilization): We remove a disk from $S$ which is disjoint from all upper and lower circles and replace it by a punctured torus with one upper and one lower (oriented) circles. One of them corresponds to the standard meridian and the other to the standard longitude of the added torus (see Figure 6$)$. The added upper circle is colored with $1 \in \pi$.

TYPE V (sliding a circle past another): Let $C_{1}$ and $C_{2}$ be two circles of a $\pi$-colored Heegaard diagram, both upper or both lower, and let $b$ be a band on $S$ which connects $C_{1}$ to $C_{2}$ (that is, $b: I \times I \rightarrow S$ is an embedding of $[0,1] \times[0,1]$ for which $\left.b(I \times I) \cap C_{i}=b(i \times I), i=1,2\right)$ but does not cross any other circle. The circle $C_{1}$ is replaced by

$$
C_{1}^{\prime}=C_{1} \#_{b} C_{2}=C_{1} \cup C_{2} \cup b(I \times \partial I) \backslash b(\partial I \times I) .
$$

The circle $C_{2}$ is replaced by a copy $C_{2}^{\prime}$ of itself which is slightly isotoped so that it has no point in common with $C_{1}^{\prime}$. The new circle $C_{1}^{\prime}$ (resp. $C_{2}^{\prime}$ ) inherits the orientation induced by $C_{1}$ (resp. $C_{2}$ ) (see Figure 7 ).

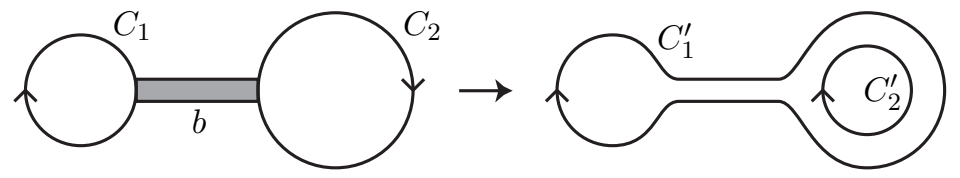

Fig. 7. Circle slide

If the two circles are both lower, then the colors of the upper circle remain unchanged. Suppose that the two circles are both upper, say $C_{1}=u_{i}$ and $C_{2}=u_{j}$ with colors $\alpha_{i}$ and $\alpha_{j}$ respectively. Set $p=b(0,1 / 2) \in u_{i}$ and $q=b(1,1 / 2) \in u_{j}$. Up to first applying a move of type II to $u_{i}$ and/or $u_{j}$, we can assume that $\left(d_{p} b(\cdot, 1 / 2), d_{p} u_{i}\right)$ is a negatively-oriented basis for $T_{p} S$ and $\left(d_{q} b(\cdot, 1 / 2), d_{q} u_{j}\right)$ is a positively-oriented basis for $T_{q} S$. Then the color of $u_{i}^{\prime}=C_{1}^{\prime}$ is $\alpha_{i}$ and the color of $u_{j}^{\prime}=C_{2}^{\prime}$ is $\alpha_{i}^{-1} \alpha_{j}$. The colors of the other upper circles remain unchanged.

One can remark that all these moves transform a $\pi$-colored Heegaard diagram into another $\pi$-colored Heegaard diagram. Indeed, for a move of type I, each word $w_{i}$ is replaced by a conjugate of itself. For a move of type II applied to an upper circle $u_{k}$, each word $w_{i}\left(x_{1}, \ldots, x_{k}, \ldots, x_{g}\right)$ is replaced by a conjugate of $w_{i}\left(x_{1}, \ldots, x_{k}^{-1}, \ldots, x_{g}\right)$. For a move of type II applied to a lower circle $l_{i}$, the word $w_{i}$ is replaced by a conjugate of $w_{i}^{-1}$. For a move of type III between $u_{k}$ and $l_{i}$, the word $w_{i}$ is replaced by a conjugate of itself in which $x_{k} x_{k}^{-1}$ or $x_{k}^{-1} x_{k}$ has been inserted. For a move of type IV, the new word $w_{g+1}\left(x_{1}, \ldots, x_{g+1}\right)$ is $x_{g+1}^{ \pm 1}$. For a move of type $\mathrm{V}$ applied to two lower circles, say $l_{i}$ slides past $l_{j}$, the word $w_{i}$ is replaced by a conjugate of itself inside which a conjugate of $w_{j}^{ \pm 1}$ has been inserted, the other words remaining unchanged (up to conjugation). For a move of type $\mathrm{V}$ applied to two upper 
circles, say $u_{i}$ slides past $u_{j}$, each word $w_{k}\left(x_{1}, \ldots, x_{j}, \ldots, x_{g}\right)$ is replaced by a conjugate of $w_{k}\left(x_{1}, \ldots, x_{i} x_{j}, \ldots, x_{g}\right)$ (see the assumptions on the orientation of the circles $u_{i}$ and $\left.u_{j}\right)$. Therefore the conditions $w_{i}\left(\alpha_{1}, \ldots, \alpha_{g}\right)=1$ are still satisfied after performing one of these moves.

2.2. Invariants of $\pi$-colored Heegaard diagrams. Fix a finite type involutory Hopf $\pi$-coalgebra $H=\left\{H_{\alpha}\right\}_{\alpha \in \pi}$ such that $\operatorname{dim} H_{1} \neq 0$ in the ground field $\mathbb{k}$ of $H$. Note that $H$ is then semisimple and cosemisimple (by Lemma 3). Using this algebraic data, we give a method to define an invariant of $\pi$-colored Heegaard diagrams, which generalizes that of Kuperberg [4].

Let $D=(S, u, l)$ be a $\pi$-colored Heegaard diagram with color $\alpha=$ $\left(\alpha_{1}, \ldots, \alpha_{g}\right)$.

To each upper circle $u_{k}$, we associate the tensor of Figure 8(a), where $c_{1}, \ldots, c_{n}$ are the crossings between $u_{k}$ and $l$ which appear in this order when making a round trip along $u_{k}$ following its orientation. Since this tensor is cyclically symmetric (see Lemma 5), this assignment does not depend on the choice of the starting point on the circle $u_{k}$ when making a round trip along it.

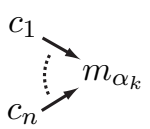

(a) Tensor associated to $u_{k}$

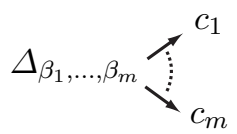

(b) Tensor associated to $l_{i}$

Fig. 8

To each lower circle $l_{i}$, we associate the tensor of Figure $8(\mathrm{~b})$, where $c_{1}, \ldots, c_{m}$ are the crossings between $l_{i}$ and $u$ which appear in this order when making a round trip along $l_{i}$ following its orientation, and the $\beta_{j} \in \pi$ are defined as follows: if $l_{i}$ intersects $u_{k}$ at $c_{j}$, then $\beta_{j}=\alpha_{k}^{\nu}$ with $\nu=+1$ if $\left(d_{c_{j}} l_{i}, d_{c_{j}} u_{k}\right)$ is a positively-oriented basis for $T_{c_{j}} S$, and $\nu=-1$ otherwise. Note that $\beta_{1} \cdots \beta_{m}=w_{i}\left(\alpha_{1}, \ldots, \alpha_{g}\right)=1$ and so the tensor associated to $l_{i}$ is well defined. Since this tensor is cyclically symmetric (see Lemma 5 ), this assignment does not depend on the choice of the starting point on the circle $l_{i}$ when making a round trip along it.

Let $c$ be a crossing point between an upper and a lower circle, say between $u_{k}$ and $l_{i}$. Let $\nu$ be as above. If $\nu=+1$, we contract the tensors assigned to $l_{i}$ and $u_{k}$ as follows:
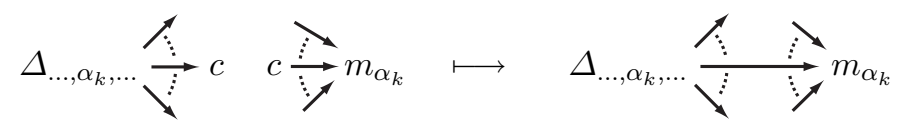
If $\nu=-1$, we contract the tensors assigned to $l_{i}$ and $u_{k}$ as follows:

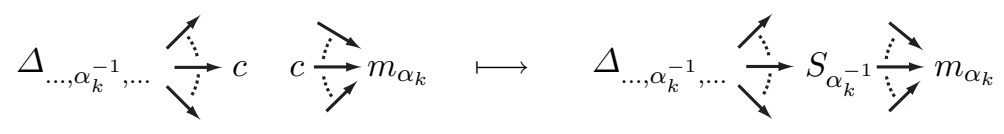

After all contractions, one gets $Z(D) \in \mathbb{k}$.

Finally, we set

$$
K_{H}(D)=\left(\operatorname{dim} H_{1}\right)^{-g} Z(D) \in \mathbb{k} .
$$

TheOREM 6. Let $H=\left\{H_{\alpha}\right\}_{\alpha \in \pi}$ be a finite type involutory Hopf $\pi$-coalgebra with $\operatorname{dim} H_{1} \neq 0$ in the ground field $\mathbb{k}$ of $H$. Then $K_{H}$ is an equivalence invariant of $\pi$-colored Heegaard diagrams.

Proof. We have to verify that $K_{H}$ is invariant under the moves of types I-V. The proof extends that of [4, Theorem 5.1] since we have to take care of the colors of the Heegaard diagrams.

Clearly, $K_{H}$ is invariant under a move of type I.

Consider a move of type II applied to an upper $u_{k}$ circle with color $\alpha_{k}$, that is, $u_{k}$ is replaced by $u_{k}^{\prime}=u_{k}$ with the opposite orientation and with color $\alpha_{k}^{-1}$. Let $c_{1}, \ldots, c_{n}$ be the crossings between $u_{k}$ and the lower circles which appear in this order following the orientation. Then the tensor associated to $u_{k}$ (resp. $u_{k}^{\prime}$ ) is:
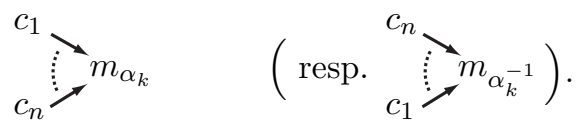

Recall that the contraction rule applied to a crossing point $c \in u_{k} \cap l_{i}$ is:

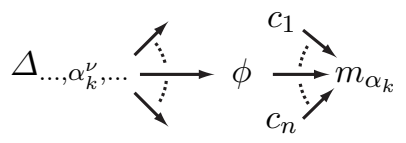

where $\nu=+1$ and $\phi=\operatorname{id}_{H_{\alpha_{k}}}$ if $\left(d_{c} l_{i}, d_{c} u_{k}\right)$ is a positively-oriented basis of $T_{c} S$, and $\nu=-1$ and $\phi=S_{\alpha_{k}^{-1}}$ otherwise. Then the contraction rule applied to the corresponding crossing point $c^{\prime} \in u_{k}^{\prime} \cap l_{i}$ is:

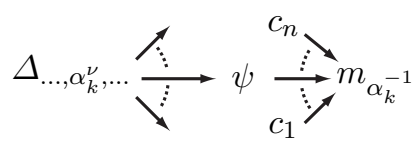

where $\psi=\operatorname{id}_{\alpha_{k}^{-1}}$ if $\left(d_{c} l_{i}, d_{c} u_{k}^{\prime}\right)$ is a positively-oriented basis of $T_{c} S$, and $\psi=S_{\alpha_{k}}$ otherwise. Now $\psi=S_{\alpha_{k}} \circ \phi$ since the antipode is involutory. 
Therefore the invariance follows from the equality

$$
c_{1} \longrightarrow S_{\alpha_{k}} S_{\alpha_{k}} m_{\alpha_{k}^{-1}}=c_{c_{n}}^{c_{1}} m_{\alpha_{k}}
$$

which comes from the anti-multiplicativity of the antipode and the fact that $T_{\alpha^{-1}} \circ S_{\alpha}=T_{\alpha}$ for any $\alpha \in \pi$ (by Lemma 4 ).

For a move of type II applied to a lower circle, the invariance follows from the equality

$$
\Delta_{\beta_{1}, \ldots, \beta_{m}} \nearrow_{c_{1}}^{c_{m}}=\Delta_{\beta_{m}^{-1}, \ldots, \beta_{1}^{-1}} \overbrace{S_{\beta_{m}^{-1}} \longrightarrow c_{m}}^{S_{\beta_{1}^{-1}} \longrightarrow c_{1}}
$$

which comes from the anti-comultiplicativity of the antipode and the fact that $S_{1}(C)=C$ (by Lemma 4$)$.

Consider now a two-point move between an upper circle with color $\alpha$ and a lower circle. Up to first applying a move of type II, we can consider that these two circles are oriented so that the invariance is a consequence of the following equality:

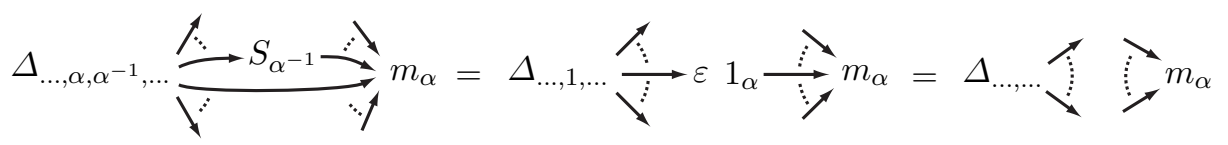

which comes from (3).

A move of type IV contributes $C \rightarrow T_{1}=\operatorname{dim} H_{1}$ (see Lemma 4) to $Z(D)$, which is canceled by normalization.

Consider a move of type $\mathrm{V}$ applied to two upper circles, say $u_{i}$ (with color $\alpha_{i}$ ) slides past $u_{j}$ (with color $\alpha_{j}$ ). We assume, as a representative case, that both circles have three crossings with the lower circles:
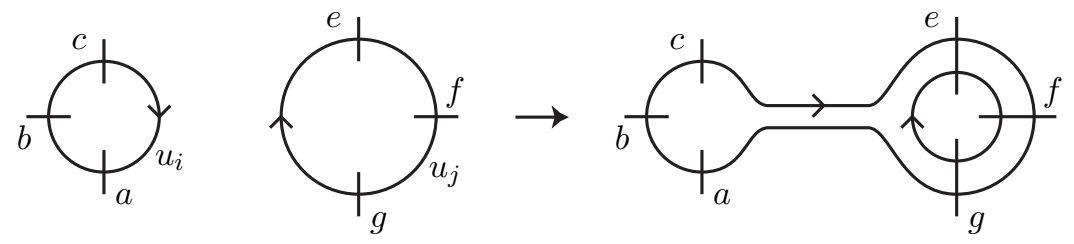

Using the anti-multiplicativity of the antipode (which allows us to consider only the positively-oriented case of the contraction rule), we see that the following factor of $Z(D)$ :

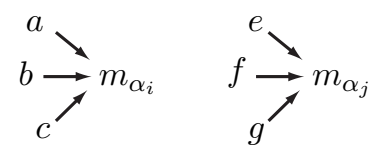


is replaced by

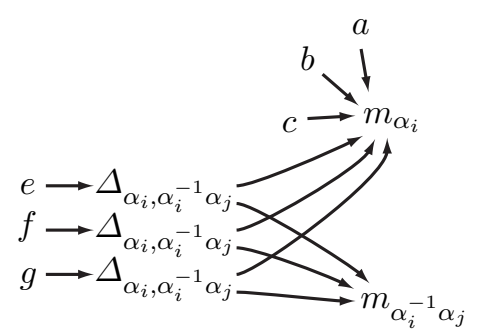

Using the multiplicativity of the comultiplication and the fact that $\left(T_{\alpha}\right)_{\alpha \in \pi}$ is a left $\pi$-integral for $H$ (see Lemma 4), we deduce that these two factors are equal (see Figure 9).

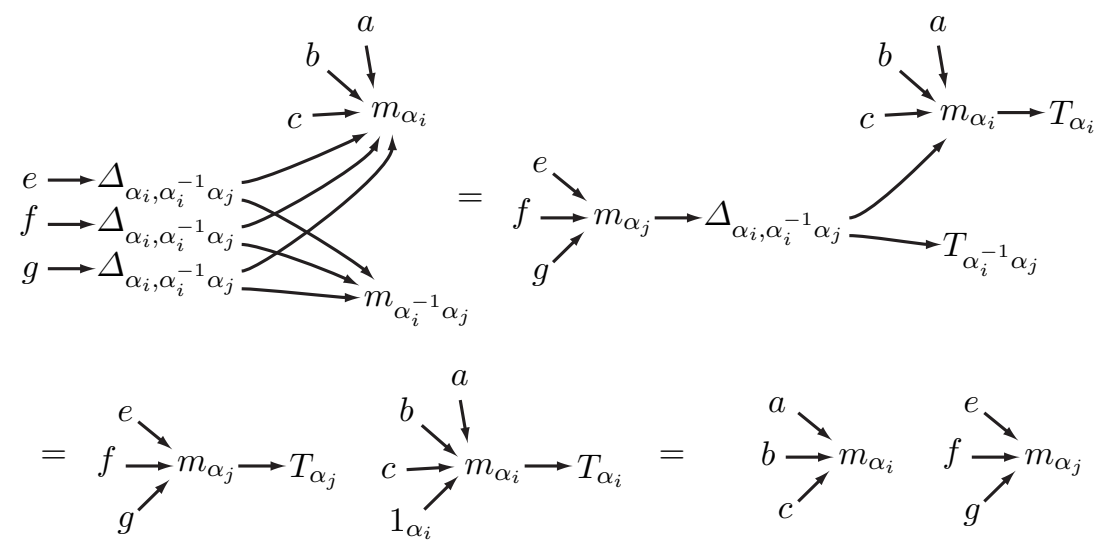

Fig. 9

Finally, suppose that a lower circle slides past another lower circle. We assume, as a representative case, that these two circles have both three crossings with the upper circles. Let $\alpha_{1}, \alpha_{2}, \alpha_{3}$ (resp. $\beta_{1}, \beta_{2}, \beta_{3}$ ) be the colors of the upper circles intersected (following the orientation) by the first (resp. second) lower circle considered. Then the invariance follows from the equality of Figure 10 which comes from the multiplicativity of the comultiplication and the fact that $C$ is a right integral for $H_{1}$ (see Lemma 4). This completes the proof of the theorem.

If $D$ is a $\pi$-colored Heegaard diagram of genus $g$ with color $\alpha=\left(\alpha_{1}, \ldots, \alpha_{g}\right)$ and $\beta \in \pi$, then $\beta \alpha \beta^{-1}=\left(\beta \alpha_{1} \beta^{-1}, \ldots, \beta \alpha_{g} \beta^{-1}\right)$ is clearly another color of the Heegaard diagram, said to be conjugate to the color $\alpha$.

Lemma 7. Let $H=\left\{H_{\alpha}\right\}_{\alpha \in \pi}$ be a finite type involutory Hopf $\pi$-coalgebra with $\operatorname{dim} H_{1} \neq 0$ in the ground field $\mathbb{k}$ of $H$. Suppose that $H=\left\{H_{\alpha}\right\}_{\alpha \in \pi}$ 


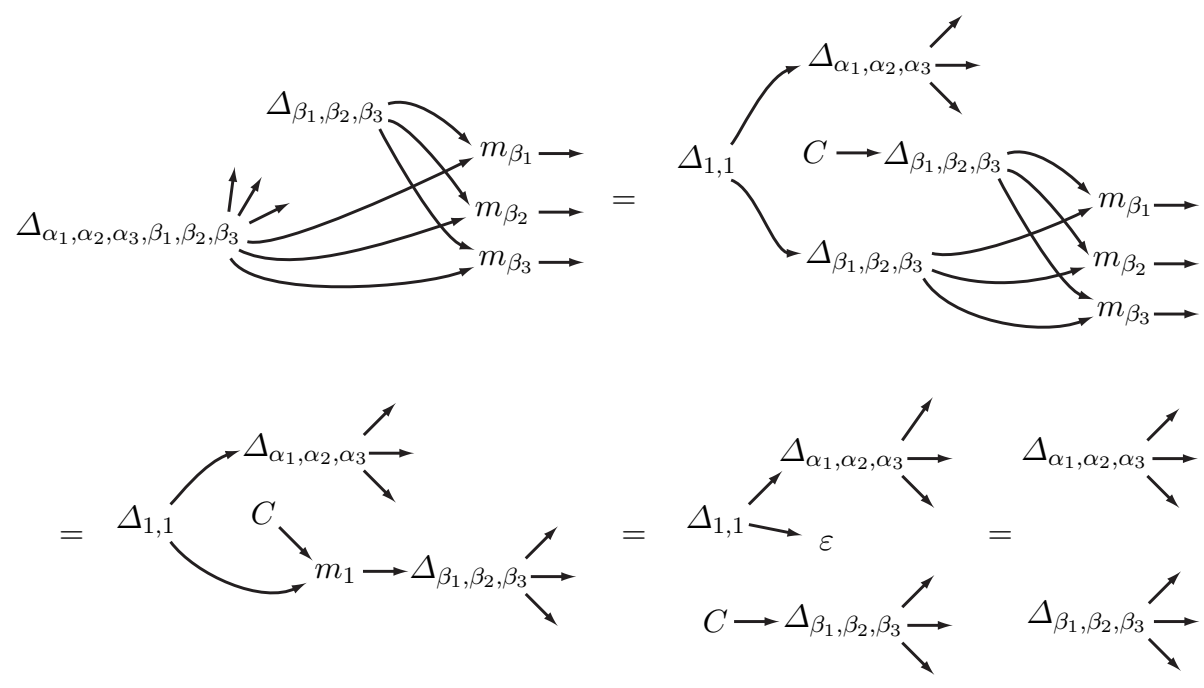

Fig. 10

is crossed (see Section 1.6). Then the invariant $K_{H}(D)$ does not depend on the conjugacy class of the color of the $\pi$-colored Heegaard diagram D.

Proof. Denote by $\varphi=\left\{\varphi_{\beta}: H_{\alpha} \rightarrow H_{\beta \alpha \beta^{-1}}\right\}_{\alpha, \beta \in \pi}$ the crossing of $H=$ $\left\{H_{\alpha}\right\}_{\alpha \in \pi}$. Let $D=(S, u, l)$ be a $\pi$-colored Heegaard diagram of genus $g$ with color $\alpha=\left(\alpha_{1}, \ldots, \alpha_{g}\right)$. Fix $\beta \in \pi$. Denote by $D^{\beta}$ the same Heegaard diagram endowed with the color $\beta \alpha \beta^{-1}=\left(\beta \alpha_{1} \beta^{-1}, \ldots, \beta \alpha_{g} \beta^{-1}\right)$. We have to verify that $K_{H}\left(D^{\beta}\right)=K_{H}(D)$.

Let $1 \leq k, i \leq g$ and denote by $c_{1}, \ldots, c_{n}$ (resp. $c_{1}^{\prime}, \ldots, c_{m}^{\prime}$ ) the crossings between $u_{k}$ and $l$ (resp. $l_{i}$ and $u$ ) which appear in this order when making a round trip along $u_{k}$ (resp. $l_{i}$ ) following its orientation. Recall that, for $D$ (resp. $D^{\beta}$ ), the tensor of Figure 11(a) (resp. Figure 11(b)) is associated to the upper circle $u_{k}$, and the tensor of Figure 11(c) (resp. Figure 11(d)) is associated to the lower circle $l_{i}$ where, if $l_{i}$ intersects some $u_{n}$ at $c_{j}^{\prime}$, then $\beta_{j}=\alpha_{n}^{\nu}$ with $\nu=1$ if $\left(d_{c_{j}^{\prime}} l_{i}, d_{c_{j}^{\prime}} u_{n}\right)$ is a positively-oriented basis for $T_{c_{j}^{\prime}} S$, and $\nu=-1$ otherwise.

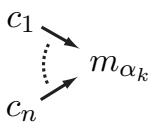

(a)

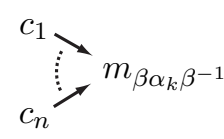

(b)

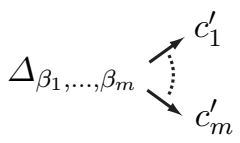

(c)

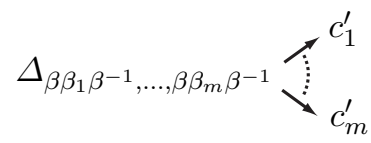

(d)

Fig. 11

Since $H$ is cosemisimple, [12, Lemmas $6.3(\mathrm{a})$ and 7.2] give that $\varphi_{\beta}(C)$ $=C$ and $[12$, Corollary 6.2 and Lemma 7.2$]$ give that $T_{\beta \alpha \beta^{-1}} \varphi_{\beta}=T_{\alpha}$ 
for all $\alpha \in \pi$. Therefore, using the multiplicativity and comultiplicativity of a crossing, we have the equalities of Figure 12.

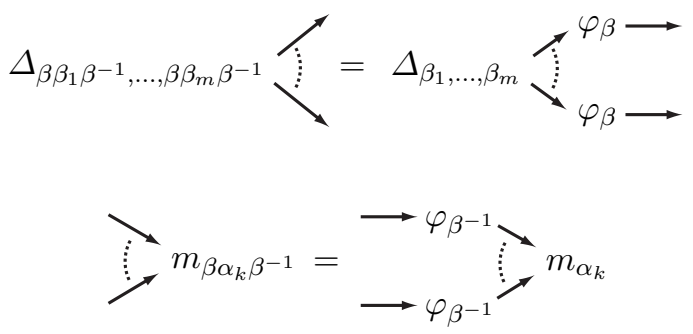

Fig. 12

Hence, since $\varphi_{\beta} \circ \varphi_{\beta^{-1}}=\operatorname{id}_{H_{\alpha}}$ and $S_{\beta \alpha \beta^{-1}} \varphi_{\beta}=\varphi_{\beta} S_{\alpha}$ for all $\alpha \in \pi$ by [12, Lemma 6.1], contracting the tensors associated to $D^{\beta}$ and $D$ leads to the same scalar $Z\left(D^{\beta}\right)=Z(D)$. Finally, $K_{H}\left(D^{\beta}\right)=\left(\operatorname{dim} H_{1}\right)^{-g} Z\left(D^{\beta}\right)=$ $\left(\operatorname{dim} H_{1}\right)^{-g} Z(D)=K_{H}(D)$.

\section{INVARIANTS OF FLAT BUNDLES OVER 3-MANIFOLDS}

In this section, we use the invariant of colored Heegaard diagrams defined in Section 2 to construct an invariant of flat bundles over 3-manifolds.

3.1. Flat bundles over 3-manifolds. Fix a group $\pi$. By a flat $\pi$-bundle over a 3-manifold, we shall mean a principal $\pi$-bundle $\xi=(p: \widetilde{M} \rightarrow M)$, where $M$ is a closed connected and oriented 3-manifold, which is flat, that is, such that its transition functions are locally constant. Such an object can be viewed as a regular covering $\widetilde{M} \rightarrow M$ with group of automorphisms $\pi$. The space $\widetilde{M}$ (resp. $M$ ) is called the total space (resp. base space) of $\xi$.

Two flat $\pi$-bundles over 3-manifolds $\xi$ and $\xi^{\prime}$ are said to be equivalent if there exists a homeomorphism $\widetilde{h}: \widetilde{M} \rightarrow \widetilde{M^{\prime}}$ which preserves the action of $\pi$ and induces an orientation-preserving homeomorphism $h: M \rightarrow M^{\prime}$.

A flat $\pi$-bundle $\xi=(p: \widetilde{M} \rightarrow M)$ is said to be pointed when its total space $\widetilde{M}$ is endowed with a base point $\widetilde{x} \in \widetilde{M}$. Two pointed flat $\pi$-bundles over 3 -manifolds $(\xi, \widetilde{x})$ and $\left(\xi^{\prime}, \widetilde{x}^{\prime}\right)$ are said to be equivalent if there exists an equivalence $\widetilde{h}: \widetilde{M} \rightarrow \widetilde{M^{\prime}}$ between them such that $\widetilde{h}(\widetilde{x})=\widetilde{x}^{\prime}$.

Let $(\xi, \widetilde{x})$ be a pointed flat $\pi$-bundle over a 3 -manifold. Set $x=p(\widetilde{x}) \in M$, where $p: \widetilde{M} \rightarrow M$ is the bundle map of $\xi$. We can associate to $(\xi, \widetilde{x})$ a morphism $f: \pi_{1}(M, x) \rightarrow \pi$, called the monodromy of $\xi$ at $\widetilde{x}$, by the following procedure: any loop $\gamma$ in $(M, x)$ uniquely lifts to a path $\widetilde{\gamma}$ in $\widetilde{M}$ beginning at $\widetilde{x}$. The path $\widetilde{\gamma}$ ends at $\alpha \cdot \widetilde{x}$ for a unique $\alpha \in \pi$. The monodromy is defined by $f([\gamma])=\alpha$, where $[\gamma]$ denotes the homotopy class in $\pi_{1}(M, x)$ of the loop $\gamma$. 
Any pointed flat $\pi$-bundle $(\xi, \widetilde{x})$ over a 3 -manifold $M$ leads to a triple $(M, x, f)$, where $x$ is the image of $\widetilde{x}$ under the bundle map $\widetilde{M} \rightarrow M$ of $\xi$ and $f: \pi_{1}(M, x) \rightarrow \pi$ is the monodromy of $\xi$ at $\widetilde{x}$. Conversely, a triple $(M, x, f)$, where $M$ is a (closed, connected, and oriented) 3 -manifold, $x \in M$, and $f: \pi_{1}(M, x) \rightarrow \pi$ is a group homomorphism, leads to a pointed flat $\pi$-bundle over $M$ uniquely determined up to equivalence (see [3, Proposition 14.1]). Let us briefly recall the construction of the pointed flat $\pi$-bundle $\xi=(p: \widetilde{M} \rightarrow M)$ associated to a triple $(M, x, f)$ : the pointed manifold $(M, x)$ admits a universal covering $u:(Y, y) \rightarrow(M, x)$. The fundamental group $\pi_{1}(M, x)$ acts on the left on $Y$ as follows: given $[\sigma] \in \pi_{1}(M, x)$ and $z \in Y$, let $y^{\prime}$ be the endpoint of the lift (in $Y$ ) of $\sigma$ to a path that starts at $y$. Choose a path $\gamma$ from $y$ to $z$ in $Y$. Then $[\sigma] \cdot z$ is defined to be the endpoint of the lift (in $Y$ ) of the path $u \circ \gamma$ that starts at $y^{\prime}$. Set $\widetilde{M}=(Y \times \pi) / \pi_{1}(M, x)$, where $\pi_{1}(M, x)$ acts on the left on $Y \times \pi$ by the rule $[\sigma] \cdot(z, \alpha)=\left([\sigma] \cdot z, \alpha f([\sigma])^{-1}\right)$. Let $\langle z, \alpha\rangle \in \widetilde{M}$ denote the image of $(z, \alpha) \in Y \times \pi$ and define $p: \widetilde{M} \rightarrow M$ by $p(\langle z, \alpha\rangle)=u(z)$. Then $\xi=(p: \widetilde{M} \rightarrow M)$ is a flat $\pi$-bundle over $M$, and it is pointed with base point $\widetilde{x}=\langle y, 1\rangle$.

It may be convenient to adopt this second point of view. In particular, under this point of view, two pointed flat $\pi$-bundles over 3 -manifolds $(M, x, f)$ and $\left(M^{\prime}, x^{\prime}, f^{\prime}\right)$ are equivalent if there exists an orientation-preserving homeomorphism $h: M \rightarrow M^{\prime}$ such that $h(x)=x^{\prime}$ and $f^{\prime} \circ h_{*}=f$, where $h_{*}: \pi_{1}(M, x) \rightarrow \pi_{1}\left(M^{\prime}, x^{\prime}\right)$ is the group isomorphism induced by $h$.

3.2. Heegaard diagrams of 3-manifolds. We first recall that a Heegaard splitting of genus $g$ of a closed, connected, and oriented 3-manifold $M$ is an ordered pair $\left(M_{u}, M_{l}\right)$ of submanifolds of $M$, both homeomorphic to a handlebody of genus $g$, such that $M=M_{u} \cup M_{l}$ and $M_{u} \cap M_{l}=\partial M_{u}=\partial M_{l}$. The handlebody $M_{u}$ (resp. $M_{l}$ ) is called upper (resp. lower) and the surface $\partial M_{u}=\partial M_{l}$ is called a Heegaard surface (of genus $g$ ) for $M$.

It is well known that every closed, connected, and oriented 3-manifold $M$ has a Heegaard splitting (e.g., by taking a closed regular neighborhood of the one-dimensional skeleton of a triangulation of $M$ and the closure of its complement).

Let $\left(M_{u}, M_{l}\right)$ be a Heegaard splitting of genus $g$ of a closed, connected, and oriented 3-manifold $M$. Since $M_{u}$ is homeomorphic to a handlebody of genus $g$, there exists a finite collection $\left\{D_{1}, \ldots, D_{g}\right\}$ of pairwise disjoint properly embedded 2-disks in $M_{u}$ which cut $M_{u}$ into a 3-ball. Likewise, there exists a finite collection $\left\{D_{1}^{\prime}, \ldots, D_{g}^{\prime}\right\}$ of pairwise disjoint properly embedded 2-disks in $M_{l}$ which cut $M_{l}$ into a 3-ball. For $1 \leq i \leq g$, set $u_{i}=\partial D_{i}$ and $l_{i}=\partial D_{i}^{\prime}$. We can (and do) suppose that these circles meet transversely. 
Denote the Heegaard surface $M_{u} \cap M_{l}$ by $S$. It is oriented as follows: for any point $p \in S$, a basis $\left(e_{1}, e_{2}\right)$ of $T_{p} S$ is positive if, when completing $\left(e_{1}, e_{2}\right)$ with a vector $e_{3}$ pointing from $M_{l}$ to $M_{u}$, we obtain a positively-oriented basis $\left(e_{1}, e_{2}, e_{3}\right)$ of $T_{p} M$. Then $D=\left(S, u=\left\{u_{1}, \ldots, u_{g}\right\}, l=\left\{l_{1}, \ldots, l_{g}\right\}\right)$ is a Heegaard diagram in the sense of Section 2.1. Such a Heegaard diagram is called a Heegaard diagram (of genus $g$ ) of $M$.

\subsection{Kuperberg-type invariants of flat bundles over 3-manifolds.} Fix a finite type involutory Hopf $\pi$-coalgebra $H=\left\{H_{\alpha}\right\}_{\alpha \in \pi}$ with $\operatorname{dim} H_{1} \neq 0$ in the ground field $\mathbb{k}$ of $H$.

Let $(\xi=(p: \widetilde{M} \rightarrow M), \widetilde{x})$ be a pointed flat $\pi$-bundle over a 3 -manifold $M$. Set $x=p(\widetilde{x}) \in M$ and let $f: \pi_{1}(M, x) \rightarrow \pi$ be the monodromy of $\xi$ at $\widetilde{x}$. Consider a Heegaard diagram $D=(S, u, l)$ of genus $g$ of $M$. Recall that $S=\partial M_{u}=\partial M_{l} \subset M$, where $\left(M_{u}, M_{l}\right)$ is a Heegaard splitting of $M$. We arbitrarily orient the upper and lower circles so that $D$ is oriented. We can (and always do) assume that $x \in S \backslash\{u, l\}$.

Since $S \backslash u$ is homeomorphic to a sphere from which $2 g$ disks have been deleted, there exist $g$ pairwise disjoint (off $x$ ) loops $\gamma_{1}, \ldots, \gamma_{g}$ on $(S, x)$ such that, for any $1 \leq i \leq g$,

- $\gamma_{i}$ intersects the upper circle $u_{i}$ in exactly one point $p_{i}$ in such a way that $\left(d_{p_{i}} \gamma_{i}, d_{p_{i}} u_{i}\right)$ is a positively-oriented basis of $T_{p_{i}} S$;

- $\gamma_{i}$ does not intersect any other upper circle.

Then the homotopy classes $a_{i}=\left[\gamma_{i}\right] \in \pi_{1}(M, x)$ do not depend on the choice of the loops $\gamma_{i}$ satisfying the above conditions (since each $\gamma_{i}$ is homotopic to a unique leaf of the $x$-based $g$-leafed rose formed by the core of the handlebody $M_{u}$ ).

Lemma 8. $\pi_{1}(M, x)=\left\langle a_{1}, \ldots, a_{g}\right| w_{j}\left(a_{1}, \ldots, a_{g}\right)=1$ for $\left.1 \leq j \leq g\right\rangle$, where the words $w_{i}\left(x_{1}, \ldots, x_{g}\right)$ are defined as in Section 2.1 .

Proof. Recall that there exists a finite collection $\left\{D_{1}, \ldots, D_{g}\right\}$ (resp. $\left\{D_{1}^{\prime}, \ldots, D_{g}^{\prime}\right\}$ ) of pairwise disjoint properly embedded 2-disks in $M_{u}$ (resp. $M_{l}$ ) which cut $M_{u}$ (resp. $M_{l}$ ) into a 3-ball. Since $\bigcup_{1 \leq i \leq g} D_{i} \cup S$ is a deformation retract of $M_{u}$ from which some 3 -balls have been deleted, $\pi_{1}\left(\bigcup_{1<i<g} D_{i}\right.$ $\cup S, x)$ is the free group generated by the homotopy classes of the loops $\gamma_{1}, \ldots, \gamma_{g}$. Now, by the Seifert-van Kampen theorem, gluing a disk $D_{j}^{\prime}$ amounts to adding the relation $w_{j}\left(\left[\gamma_{1}\right], \ldots,\left[\gamma_{g}\right]\right)=1$. Hence the lemma follows from the fact that $\bigcup_{1 \leq i \leq g}\left(D_{i} \cup D_{i}^{\prime}\right) \cup S$ is a deformation retract of $M$ from which some 3-balls have been deleted.

For any $1 \leq i \leq g$, set $\alpha_{i}=f\left(a_{i}\right) \in \pi$. By Lemma $8, \alpha=\left(\alpha_{1}, \ldots, \alpha_{g}\right)$ is a color of the oriented Heegaard diagram $D$. We say that the (oriented) Heegaard diagram $D$ of $M$ is colored by $f$. 
Finally, we set

$$
K_{H}(\xi, \widetilde{x})=K_{H}(D) \in \mathbb{k},
$$

where $K_{H}$ is the invariant of $\pi$-colored Heegaard diagrams of Theorem 6 .

TheORem 9. Let $H=\left\{H_{\alpha}\right\}_{\alpha \in \pi}$ be a finite type involutory Hopf $\pi$-coalgebra with $\operatorname{dim} H_{1} \neq 0$ in the ground field $\mathbb{k}$ of $H$.

(a) $K_{H}$ is an invariant of pointed flat $\pi$-bundles over 3-manifolds.

(b) Let $\xi=(p: \widetilde{M} \rightarrow M)$ be a flat $\pi$-bundle over a 3-manifold $M$.

(i) The function $\widetilde{x} \in \widetilde{M} \mapsto K_{H}(\xi, \widetilde{x}) \in \mathbb{k}$ is constant on the pathconnected components of $\widetilde{M}$;

(ii) If $H$ is crossed or if $\pi$ is abelian or if the monodromy of $\xi$ is surjective, then $K_{H}(\xi, \widetilde{x})$ does not depend on the choice of the base point $\widetilde{x} \in \widetilde{M}$.

This theorem is proved in Section 3.5.

If $\pi=1$ and $M$ is a 3-manifold, then $K_{H}\left(\mathrm{id}_{M}: M \rightarrow M\right)$ coincides with the invariant of $M$ constructed by Kuperberg [4].

In Section 4, we give examples which show that the invariant $K_{H}$ is non-trivial.

3.4. Basic properties of $K_{H}$. Let $H=\left\{H_{\alpha}\right\}_{\alpha \in \pi}$ be a finite type involutory Hopf $\pi$-coalgebra with $\operatorname{dim} H_{1} \neq 0$ in the ground field $\mathbb{k}$ of $H$. Recall that $H^{\mathrm{op}}$ and $H^{\mathrm{cop}}$ denote the Hopf $\pi$-coalgebras opposite and coopposite to $H$ (see Section 1.1).

Let $(\xi, \widetilde{x})$ be a pointed flat $\pi$-bundle over a 3 -manifold $M$. Denote by $-\xi$ the flat $\pi$-bundle $\xi$ whose base space $M$ is endowed with the opposite orientation. Then

$$
K_{H}(-\xi, \widetilde{x})=K_{H^{\mathrm{cop}}}(\xi, \widetilde{x})=K_{H^{\mathrm{op}}}(\xi, \widetilde{x}) .
$$

Indeed, starting from an oriented Heegaard diagram $D=(S, u, l)$ for $M$, reversing the orientation of $M$ amounts to reversing the orientation of the Heegaard surface $S$. Then the first equality of (5) can be easily obtained by reversing the orientation of the lower circles, and the second one by reversing the orientation of the upper circles.

Let $\left(\xi_{1}, \widetilde{x}_{1}\right)$ and $\left(\xi_{2}, \widetilde{x}_{2}\right)$ be two pointed flat $\pi$-bundles over 3 -manifolds. Denote by $x_{1}$ (resp. $\left.x_{2}\right)$ the image of $\widetilde{x}_{1}$ (resp. $\widetilde{x}_{2}$ ) under the bundle map $\widetilde{M}_{1} \rightarrow M_{1}$ of $\xi_{1}$ (resp. $\widetilde{M}_{2} \rightarrow M_{2}$ of $\xi_{2}$ ), and by $f_{1}: \pi_{1}\left(M_{1}\right) \rightarrow \pi$ (resp. $\left.f_{2}: \pi_{1}\left(M_{2}\right) \rightarrow \pi\right)$ the monodromy of $\xi_{1}$ at $\widetilde{x}_{1}$ (resp. of $\xi_{2}$ at $\widetilde{x}_{2}$ ). Take closed 3-balls $B_{1} \subset M_{1}$ and $B_{2} \subset M_{2}$ such that $x_{1} \in \partial B_{1}$ and $x_{2} \in \partial B_{2}$. Glue $M_{1} \backslash \operatorname{Int} B_{1}$ and $M_{2} \backslash \operatorname{Int} B_{2}$ along a homeomorphism $h: \partial B_{1} \rightarrow \partial B_{2}$ chosen so that $h\left(x_{1}\right)=x_{2}$ and that the orientations in $M_{1} \backslash \operatorname{Int} B_{1}$ and $M_{2} \backslash$ Int $B_{2}$ induced by those in $M_{1}, M_{2}$ are compatible. This gluing yields 
a closed, connected, and oriented 3-manifold $M_{1} \# M_{2}$. For $i=1$ or 2 , consider the embeddings $j_{i}: M_{i} \backslash \operatorname{Int} B_{i} \hookrightarrow M_{i}$ and $k_{i}: M_{i} \backslash \operatorname{Int} B_{i} \hookrightarrow M_{1} \# M_{2}$ and set $x=k_{1}\left(x_{1}\right)=k_{2}\left(x_{2}\right)$. By the van Kampen theorem, since $\partial B_{2} \cong$ $h\left(\partial B_{1}\right)$ is simply-connected, there exists a unique group homomorphism $f: \pi_{1}\left(M_{1} \# M_{2}, x\right) \rightarrow \pi$ such that $f \circ\left(k_{i}\right)_{*}=f_{i} \circ\left(j_{i}\right)_{*}(i=1,2)$. This leads to a triple $\left(M_{1} \# M_{2}, x, f\right)$, and so to a pointed flat $\pi$-bundle over $M_{1} \# M_{2}$, denoted by $\left(\xi_{1} \# \xi_{2}, \widetilde{x}\right)$, whose monodromy is $f$ (see Section 3.1). Then

$$
K_{H}\left(\xi_{1} \# \xi_{2}, \widetilde{x}\right)=K_{H}\left(\xi_{1}, \widetilde{x}_{1}\right) K_{H}\left(\xi_{2}, \widetilde{x}_{2}\right) .
$$

Indeed, we can choose a Heegaard diagram for $M$ which is a connected sum of Heegaard diagrams for $M_{1}$ and $M_{2}$ and such that the colorations of these diagrams by the monodromies $f, f_{1}$, or $f_{2}$ are compatible with this connected sum.

3.5. Proof of Theorem 9. To prove (a), adopting the second point of view of Section 3.1, let $(M, x, f)$ and $\left(M^{\prime}, x^{\prime}, f^{\prime}\right)$ be two equivalent pointed flat $\pi$-bundles over 3-manifolds. Choose Heegaard diagrams $D=(S, u, l)$ of genus $g$ of $M$ and $D^{\prime}=\left(S^{\prime}, u^{\prime}, l^{\prime}\right)$ of genus $g^{\prime}$ of $M^{\prime}$. Let $h: M \rightarrow M^{\prime}$ be an orientation-preserving homeomorphism with $h(x)=x^{\prime}$ and $f=$ $f^{\prime} \circ h_{*}$, where $h_{*}: \pi_{1}(M, x) \rightarrow \pi_{1}\left(M^{\prime}, x^{\prime}\right)$ is the homomorphism induced by $h$ in homotopy. By the Reidemeister-Singer theorem (see [5, Theorem 8] or [4, Theorem 4.1]), we can restrict ourselves to the case where the diagrams $D^{\prime}$ and $D$ are related by a move (or its inverse) of the following type:

- Type A: $S^{\prime}=h(S), u^{\prime}=h(u)$, and $l^{\prime}=h(l)$;

- Type B: $S^{\prime}=h(S), u^{\prime}=h(u)$, and $l^{\prime}$ is isotopic to $h(l)$ relative to $u^{\prime}$;

- TYpe C: $S^{\prime}=h(S) \# T^{2}, u^{\prime}=h(u) \cup\left\{C_{1}\right\}$, and $l^{\prime}=h(l) \cup\left\{C_{2}\right\}$, where $T^{2}$ is a torus and $\left\{C_{1}, C_{2}\right\}$ is the set formed by the standard meridian and longitude of $T^{2}$;

- Type D: $S^{\prime}=h(S), u^{\prime}=h(u)$, and $l^{\prime}$ is obtained from $h(l)$ by sliding one circle of $h(l)$ past another circle of $h(l)$, avoiding the other upper and lower circles of $h(S)$;

- Type E: $S^{\prime}=h(S), l^{\prime}=h(l)$, and $u^{\prime}$ is obtained from $h(u)$ by sliding one circle of $h(u)$ past another circle of $h(u)$, avoiding the other upper and lower circles of $h(S)$.

Without loss of generality, we can assume that $x \in S \backslash\{u, l\}$ and $x^{\prime} \in$ $S^{\prime} \backslash\left\{u^{\prime}, l^{\prime}\right\}$. We arbitrarily orient the upper circles and lower circles of $D$ and $D^{\prime}$. Denote by $\alpha=\left(\alpha_{1}, \ldots, \alpha_{g}\right)$ the coloration of the diagram $D$ by the homomorphism $f$, and by $\alpha^{\prime}=\left(\alpha_{1}^{\prime}, \ldots, \alpha_{g^{\prime}}^{\prime}\right)$ the coloration of the diagram $D^{\prime}$ by the homomorphism $f^{\prime}=f \circ h_{*}^{-1}$. 
By Theorem 6, it suffices to prove that the $\pi$-colored Heegaard diagrams $D$ and $D^{\prime}$ are equivalent, i.e., that they are related by a finite sequence of moves of types I-V (or their inverses) described in Section 2.1.

Note that, up to applying some moves of type II, we can assume that the orientations of the upper and lower circles are transported by the homeomorphisms $h$. Indeed, if we change the orientation of an upper circle $u_{i}$ to its inverse, then the color $\alpha_{i}=f\left(\left[\gamma_{i}\right]\right)$ is replaced by $f\left(\left[\gamma_{i}^{-1}\right]\right)=\alpha_{i}^{-1}$, where $\gamma_{i}$ is a loop on $(S, x)$ which crosses (in a positively-oriented way) the upper circle $u_{i}$ in exactly one point and does not intersect any other upper circle.

Suppose that $D^{\prime}$ is obtained from $D$ by a move of type A. Let $1 \leq i \leq g$ and $\gamma_{i}$ be a loop on $(S, x)$ which crosses (in a positively-oriented way) the upper circle $u_{i}$ in exactly one point and does not intersect any other upper circle of $D$. Then $\gamma_{i}^{\prime}=h\left(\gamma_{i}\right)$ is a loop on $\left(S^{\prime}, x^{\prime}\right)$ which crosses (in a positivelyoriented way) the upper circle $u_{i}^{\prime}=h\left(u_{i}\right)$ in exactly one point and does not intersect any other upper circle of $D^{\prime}$. Therefore

$$
\alpha_{i}^{\prime}=f^{\prime}\left(\left[\gamma_{i}^{\prime}\right]\right)=f^{\prime}\left(\left[h\left(\gamma_{i}\right)\right]\right)=f^{\prime} \circ h_{*}\left(\left[\gamma_{i}\right]\right)=f\left(\left[\gamma_{i}\right]\right)=\alpha_{i} .
$$

Hence the $\pi$-colored Heegaard diagrams $D^{\prime}$ and $D$ are related by a move of type I.

Suppose that $D^{\prime}$ is obtained from $D$ by a move of type $\mathrm{B}$. Then the colors of the upper circles $u_{i}^{\prime}$ and $u_{i}$ agree (by the same argument as above). Therefore the $\pi$-colored Heegaard diagram $D^{\prime}$ is obtained from the $\pi$-colored Heegaard diagram $D$ by a finite sequence of moves of type I and III (by decomposing the isotopy into two-point moves, see Section 2.1).

Suppose that $D^{\prime}$ is obtained from $D$ by a move of type C. Since $u^{\prime}=$ $h(u) \cup\left\{C_{1}\right\}$, the color of the upper circle $u_{i}=h\left(u_{i}\right)$ agrees with that of the upper circle $u_{i}$ for any $1 \leq i \leq g=g^{\prime}-1$. Let $\varrho$ be a path connecting the point $x^{\prime}$ to the circle $C_{2}$ which does not intersect any upper circle of $D^{\prime}$. Then the loop $\varrho C_{2} \varrho^{-1}$ crosses $C_{1}$ in exactly one point and does not intersect any other upper circle of $D^{\prime}$. Set $\nu=+1$ if $\varrho C_{2} \varrho^{-1}$ crosses $C_{1}$ in a positivelyoriented way, and $\nu=-1$ otherwise. Therefore

$$
\alpha_{g^{\prime}}^{\prime}=f^{\prime}\left(\left[\varrho C_{2}^{\nu} \varrho^{-1}\right]\right)=f^{\prime}\left(\left[\varrho C_{2} \varrho^{-1}\right]\right)^{\nu} .
$$

Now the circle $C_{2}$ is contractible in $M^{\prime}$. Thus $\left[\varrho C_{2} \varrho^{-1}\right]=1 \in \pi_{1}\left(M^{\prime}, x^{\prime}\right)$ and so $\alpha_{g^{\prime}}^{\prime}=1 \in \pi$. Hence the $\pi$-colored Heegaard diagram $D^{\prime}$ is obtained from the $\pi$-colored Heegaard diagram $D$ by a move of type I followed by a move of type IV.

Suppose that $D^{\prime}$ is obtained from $D$ by a move of type D. Since $S^{\prime}=h(S)$ and $u^{\prime}=h(u)$, the colors of the upper circles of $D^{\prime}$ and $D$ agree. Then the $\pi$-colored Heegaard diagram $D^{\prime}$ is obtained from the $\pi$-colored Heegaard diagram $D$ by a move of type I followed by a move of type $\mathrm{V}$. 


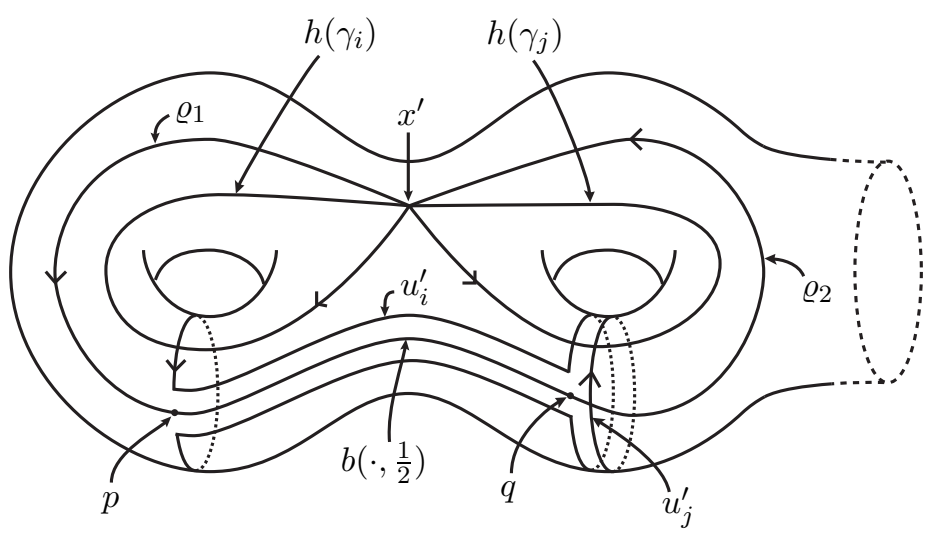

Fig. 13

Finally, suppose that $D^{\prime}$ is obtained from $D$ by a move of type E, i.e., $u^{\prime}$ is obtained from $h(u)$ by sliding a circle $h\left(u_{i}\right)$ past another circle $h\left(u_{j}\right)$. Let $b: I \times I \rightarrow S^{\prime}$ be a band which connects $h\left(u_{i}\right)$ to $h\left(u_{j}\right)$ (that is, $b(I \times I)$ $\cap h\left(u_{i}\right)=b(0 \times I)$ and $\left.b(I \times I) \cap h\left(u_{j}\right)=b(1 \times I)\right)$ but does not intersect any other circle. We can also assume that $x^{\prime} \notin b(I \times I)$. Then

$$
u_{i}^{\prime}=h\left(u_{i}\right) \#_{b} h\left(u_{j}\right)=h\left(u_{i}\right) \cup h\left(u_{j}\right) \cup b(I \times \partial I) \backslash b(\partial I \times I)
$$

and $u_{j}^{\prime}$ is a copy $h\left(u_{j}\right)$ which is slightly isotoped so that it has no point in common with $u_{i}^{\prime}$. Set $p=b(0,1 / 2) \in h\left(u_{i}\right)$ and $q=b(1,1 / 2) \in h\left(u_{j}\right)$. Up to first applying a move of type II to $u_{i}$ and/or $u_{j}$, we can assume that the basis $\left(d_{p} b(\cdot, 1 / 2), d_{p} h\left(u_{i}\right)\right)$ for $T_{p} S^{\prime}$ is negatively-oriented and the basis $\left(d_{q} b(\cdot, 1 / 2), d_{q} h\left(u_{j}\right)\right)$ for $T_{q} S^{\prime}$ is positively-oriented. Then the orientations of $u_{i}^{\prime}$ induced by $h\left(u_{i}\right)$ and $h\left(u_{j}\right)$ respectively coincide, and $u_{i}^{\prime}$ is endowed with this orientation. Let $\gamma_{i}$ (resp. $\gamma_{j}$ ) be a loop on $(S, x)$ which crosses (in a positively-oriented way) the upper circle $u_{i}$ (resp. $u_{j}$ ) in exactly one point and does not intersect any other upper circle of $D$ nor the band $h^{-1}(b(I \times I))$. Let $\varrho_{1}: I \rightarrow S^{\prime}$ be a path with $\varrho_{1}(0)=x^{\prime}$ and $\varrho_{1}(1)=p$ which does not intersect any upper circle of $D^{\prime}$ and is such that $\left(d_{p} \varrho_{1}, d_{p} h\left(u_{i}\right)\right)$ is a negatively-oriented basis for $T_{p} S^{\prime}$. Let $\varrho_{2}: I \rightarrow S^{\prime}$ be a path with $\varrho_{2}(0)=q$ and $\varrho_{2}(1)=x^{\prime}$ which does not intersect any upper circle of $D^{\prime}$ and is such that $\left(d_{q} \varrho_{2}, d_{q} h\left(u_{j}\right)\right)$ is a positively-oriented basis for $T_{q} S^{\prime}$ (see Figure 13). Set $\gamma_{i}^{\prime}=h\left(\gamma_{i}\right)$ and $\gamma_{j}^{\prime}=\varrho_{1} b(\cdot, 1 / 2) \varrho_{2}$. They are loops on $\left(S^{\prime}, x^{\prime}\right)$. The loop $\gamma_{i}^{\prime}$ (resp. $\gamma_{j}^{\prime}$ ) crosses in a positively-oriented way the upper circle $u_{i}^{\prime}$ (resp. $u_{j}^{\prime}$ ) in exactly one point and does not intersect any other upper circle of $D^{\prime}$. Therefore

$$
\alpha_{i}^{\prime}=f^{\prime}\left(\left[\gamma_{i}^{\prime}\right]\right)=f^{\prime}\left(\left[h\left(\gamma_{i}\right)\right]\right)=f^{\prime} \circ h_{*}\left(\left[\gamma_{i}\right]\right)=f\left(\left[\gamma_{i}\right]\right)=\alpha_{i},
$$


and, since $\gamma_{j}^{\prime}$ is homotopic (in $M^{\prime}$ ) to the loop $h\left(\gamma_{i}\right)^{-1} h\left(\gamma_{j}\right)$,

$$
\alpha_{j}^{\prime}=f^{\prime}\left(\left[h\left(\gamma_{i}\right)^{-1} h\left(\gamma_{j}\right)\right]\right)=f^{\prime} \circ h_{*}\left(\left[\left(\gamma_{i}\right)^{-1} \gamma_{j}\right]\right)=f\left(\left[\gamma_{i}\right]^{-1}\left[\gamma_{j}\right]\right)=\alpha_{i}^{-1} \alpha_{j} .
$$

Hence the $\pi$-colored Heegaard diagram $D^{\prime}$ is obtained from the $\pi$-colored Heegaard diagram $D$ by a move of type I followed by a move of type V. This completes the proof of (a).

To show (b)(i) of Theorem 9 , let $\xi=(p: \widetilde{M} \rightarrow M)$ be a flat $\pi$-bundle over a 3-manifold and $\widetilde{x}, \widetilde{x}^{\prime}$ be two points in $\widetilde{M}$ which belong to the same pathcomponent. Consider a path $\widetilde{\gamma}$ in $\widetilde{M}$ connecting $\widetilde{x}$ to $\widetilde{x}^{\prime}$. Pushing $\widetilde{x}$ to $\widetilde{x}^{\prime}$ along $\widetilde{\gamma}$ inside a tubular neighborhood of $\operatorname{Im}(\widetilde{\gamma})$ in $\widetilde{M}$ yields a self-homeomorphism of $\widetilde{M}$ which is an equivalence between the pointed flat $\pi$-bundles $(\xi, \widetilde{x})$ and $\left(\xi, \widetilde{x}^{\prime}\right)$. Therefore $K_{H}(\xi, \widetilde{x})=K_{H}\left(\xi, \widetilde{x}^{\prime}\right)$ by (a).

To prove (b)(ii), fix a flat $\pi$-bundle $\xi=(p: \widetilde{M} \rightarrow M)$ over a 3-manifold $M$. Let $\widetilde{x}, \widetilde{x}^{\prime}$ be two points in $\widetilde{M}$. Set $x=p(\widetilde{x})$ and $x^{\prime}=p\left(\widetilde{x}^{\prime}\right)$. Since $M$ is connected, there exists a path $\gamma$ in $M$ connecting $x$ to $x^{\prime}$. Let $\widetilde{z} \in \widetilde{M}$ be the endpoint of the lift of $\gamma$ to a path that starts at $\widetilde{x}$. Since $p(\widetilde{z})=x^{\prime}=p\left(\widetilde{x}^{\prime}\right)$, there exists $\beta \in \pi$ such that $\widetilde{x}^{\prime}=\beta \cdot \widetilde{z}$.

Firstly, suppose that the monodromy $f$ of $\xi$ at $\widetilde{z}$ is surjective. Then there exists a loop $\sigma$ based at $x^{\prime}$ such that $f([\sigma])=\beta$. Denote by $\widetilde{\sigma}$ the lift of $\sigma$ to a path that starts at $\widetilde{z}$. Since $\widetilde{x}^{\prime}=\beta \cdot \widetilde{z}=f([\sigma]) \cdot \widetilde{z}$, the path $\widetilde{\sigma}$ ends at $\widetilde{x}^{\prime}$. Finally, $\widetilde{\gamma} \widetilde{\sigma}$ is a path in $\widetilde{M}$ which connects $\widetilde{x}$ to $\widetilde{x}^{\prime}$. Hence $K_{H}(\xi, \widetilde{x})=K_{H}\left(\xi, \widetilde{x}^{\prime}\right)$ by (b)(i).

Secondly, suppose that the Hopf $\pi$-coalgebra $H$ is crossed (see Section 1.6). Since $\widetilde{x}^{\prime}=\beta \cdot \widetilde{z}$, the monodromies $f_{\widetilde{z}}, f_{\widetilde{x}^{\prime}}: \pi_{1}\left(M, x^{\prime}\right) \rightarrow \pi$ of $\xi$ at $\widetilde{z}$ and $\widetilde{x}^{\prime}$ are related by $f_{\widetilde{x}^{\prime}}=\beta f_{\widetilde{z}} \beta^{-1}$. Let $D=(S, u, l)$ be a Heegaard diagram of genus $g$ of $M$ whose upper and lower circles are arbitrarily oriented. Denote by $D_{f_{\tilde{z}}}$ and $D_{f_{\widetilde{x}^{\prime}}}$ the $\pi$-colored Heegaard diagrams obtained by coloring $D$ with $f_{\widetilde{z}}$ and $f_{\widetilde{x}^{\prime}}$. Since $f_{\widetilde{x}^{\prime}}=\beta f_{\widetilde{z}} \beta^{-1}$, the colors of $D_{f_{\tilde{z}}}$ and $D_{f_{\tilde{x}^{\prime}}}$ are conjugate. Therefore, by using Lemma 7 and then (b)(i), we get

$$
K_{H}\left(\xi, \widetilde{x}^{\prime}\right)=K_{H}\left(D_{f_{\widetilde{x}^{\prime}}}\right)=K_{H}\left(D_{f_{\tilde{z}}}\right)=K_{H}(\xi, \widetilde{z})=K_{H}(\xi, \widetilde{x}) .
$$

Finally, suppose that $\pi$ is abelian. Then the Hopf $\pi$-coalgebra $H=$ $\left\{H_{\alpha}\right\}_{\alpha \in \pi}$ is crossed (see Section 1.6) and so $K_{H}(\xi, \widetilde{x})=K_{H}\left(\xi, \widetilde{x}^{\prime}\right)$ by the previous case. This completes the proof of Theorem 9 .

\section{EXAMPLES}

In this section, we give some examples of computations of the scalar invariant of flat bundles over 3-manifolds constructed in Section 3. Although rather elementary, the Hopf group-coalgebras that we use here are sufficient 
to show that the invariant constructed in Section 3 is non-trivial. For more elaborated examples of Hopf group-coalgebras, we refer to [13].

4.1. Example 1. As remarked by Vainerman [10], the Kac-Paljutkin Hopf algebra $A=\mathbb{C}^{4} \oplus \operatorname{Mat}_{2}(\mathbb{C})$, viewed as a central prolongation of $F(\mathbb{Z} / 2 \mathbb{Z})$, leads to a finite type involutory Hopf $\mathbb{Z} / 2 \mathbb{Z}$-coalgebra $H=\left\{H_{0}, H_{1}\right\}$ over $\mathbb{C}$. Namely, set $H_{0}=\mathbb{C}^{4}$ and $H_{1}=\operatorname{Mat}_{2}(\mathbb{C})$ as algebras. Let $\left\{e_{1}, e_{2}, e_{3}, e_{4}\right\}$ be the (standard) basis of $H_{0}$ and $\left\{e_{1,1}, e_{1,2}, e_{2,1}, e_{2,2}\right\}$ be the (standard) basis of $H_{1}$. The counit $\varepsilon: H_{0} \rightarrow \mathbb{C}$ is given by $\varepsilon\left(e_{1}\right)=1$ and $\varepsilon\left(e_{2}\right)=\varepsilon\left(e_{3}\right)=$ $\varepsilon\left(e_{4}\right)=0$. The comultiplication is given by

$$
\begin{gathered}
\Delta_{0,0}\left(e_{1}\right)=e_{1} \otimes e_{1}+e_{2} \otimes e_{2}+e_{3} \otimes e_{3}+e_{4} \otimes e_{4}, \\
\Delta_{0,0}\left(e_{2}\right)=e_{1} \otimes e_{2}+e_{2} \otimes e_{1}+e_{3} \otimes e_{4}+e_{4} \otimes e_{3}, \\
\Delta_{0,0}\left(e_{3}\right)=e_{1} \otimes e_{3}+e_{3} \otimes e_{1}+e_{2} \otimes e_{4}+e_{4} \otimes e_{2}, \\
\Delta_{0,0}\left(e_{4}\right)=e_{1} \otimes e_{4}+e_{4} \otimes e_{1}+e_{2} \otimes e_{3}+e_{3} \otimes e_{2}, \\
\Delta_{0,1}\left(e_{1,1}\right)=e_{1} \otimes e_{1,1}+e_{2} \otimes e_{2,2}+e_{3} \otimes e_{1,1}+e_{4} \otimes e_{2,2}, \\
\Delta_{0,1}\left(e_{1,2}\right)=e_{1} \otimes e_{1,2}-i e_{2} \otimes e_{2,1}-e_{3} \otimes e_{1,2}+i e_{4} \otimes e_{2,1}, \\
\Delta_{0,1}\left(e_{2,1}\right)=e_{1} \otimes e_{2,1}+i e_{2} \otimes e_{1,2}-e_{3} \otimes e_{2,1}-i e_{4} \otimes e_{1,2}, \\
\Delta_{0,1}\left(e_{2,2}\right)=e_{1} \otimes e_{2,2}+e_{2} \otimes e_{1,1}+e_{3} \otimes e_{2,2}+e_{4} \otimes e_{1,1}, \\
\Delta_{1,0}\left(e_{1,1}\right)=e_{1,1} \otimes e_{1}+e_{2,2} \otimes e_{2}+e_{1,1} \otimes e_{3}+e_{2,2} \otimes e_{4}, \\
\Delta_{1,0}\left(e_{1,2}\right)=e_{1,2} \otimes e_{1}+i e_{2,1} \otimes e_{2}-e_{1,2} \otimes e_{3}-i e_{2,1} \otimes e_{4}, \\
\Delta_{1,0}\left(e_{2,1}\right)=e_{2,1} \otimes e_{1}-i e_{1,2} \otimes e_{2}-e_{2,1} \otimes e_{3}+i e_{1,2} \otimes e_{4}, \\
\Delta_{1,0}\left(e_{2,2}\right)=e_{2,2} \otimes e_{1}+e_{1,1} \otimes e_{2}+e_{2,2} \otimes e_{3}+e_{1,1} \otimes e_{4}, \\
\Delta_{1,1}\left(e_{1}\right)=\frac{1}{2}\left(e_{1,1} \otimes e_{1,1}+e_{2,2} \otimes e_{2,2}+e_{1,2} \otimes e_{1,2}+e_{2,1} \otimes e_{2,1}\right), \\
\Delta_{1,1}\left(e_{2}\right)=\frac{1}{2}\left(e_{1,1} \otimes e_{2,2}+e_{2,2} \otimes e_{1,1}+i e_{1,2} \otimes e_{2,1}-i e_{2,1} \otimes e_{1,2}\right), \\
\Delta_{1,1}\left(e_{3}\right)=\frac{1}{2}\left(e_{1,1} \otimes e_{1,1}+e_{2,2} \otimes e_{2,2}-e_{1,2} \otimes e_{1,2}-e_{2,1} \otimes e_{2,1}\right), \\
\Delta_{1,1}\left(e_{4}\right)=\frac{1}{2}\left(e_{1,1} \otimes e_{2,2}+e_{2,2} \otimes e_{1,1}-i e_{1,2} \otimes e_{2,1}+i e_{2,1} \otimes e_{1,2}\right) .
\end{gathered}
$$

The antipode is given by $S_{0}\left(e_{k}\right)=e_{k}$ for any $1 \leq k \leq 4$ and $S_{1}\left(e_{k, l}\right)=e_{l, k}$ for any $1 \leq k, l \leq 2$.

Let $n \geq 1$. There exists two flat $\mathbb{Z} / 2 \mathbb{Z}$-bundles $\xi_{n}^{0}$ and $\xi_{n}^{1}$ over the lens space $L(2 n, 1)$, whose monodromies $f_{n}^{0}, f_{n}^{1}: \pi_{1}(L(2 n, 1)) \cong \mathbb{Z} / 2 n \mathbb{Z} \rightarrow \mathbb{Z} / 2 \mathbb{Z}$ are respectively given by $f_{n}^{0}(1)=0$ and $f_{n}^{1}(1)=1$.

A Heegaard diagram $\left\{u_{1}, l_{1}\right\}$ of genus 1 of the lens space $L(2 n, 1)$ is given, on the torus $\mathbb{T}=\mathbb{R}^{2} / \mathbb{Z}^{2}$, by $u_{1}=\mathbb{R}(0,1)+\mathbb{Z}^{2}$ and $l_{1}=\mathbb{R}(1,1 / 2 n)+\mathbb{Z}^{2}$. Fix $k=0,1$ and set $\alpha=f_{n}^{k}(1) \in \mathbb{Z} / 2 \mathbb{Z}$. Denote by $D_{\alpha}$ the $\pi$-colored Heegaard diagram obtained from $\left(\mathbb{T},\left\{u_{1}, l_{1}\right\}\right)$ by providing the circle $u_{1}$ 
with the color $\alpha$. Then

$$
K_{H}\left(\xi_{n}^{k}\right)=\left(\operatorname{dim} H_{0}\right)^{-1} K_{H}\left(D_{\alpha}\right)=\frac{1}{4} K_{H}\left(D_{\alpha}\right),
$$

where $K_{H}\left(D_{\alpha}\right) \in \mathbb{C}$ equals the tensor depicted in Figure 14(a).

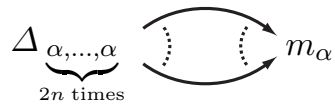

(a) $K_{H}\left(D_{\alpha}\right)$

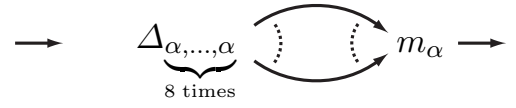

(b) $F_{\alpha}: H_{0} \rightarrow H_{\alpha}$

Fig. 14

Let $F_{\alpha}: H_{0} \rightarrow H_{\alpha}$ be the map defined in Figure 14(b). One easily checks by hand that $F_{\alpha}(x)=\varepsilon(x) 1_{\alpha}$ for all $x \in H_{0}$. Then, using the (co)associativity of the (co)multiplication, we get the equalities of Figure 15.

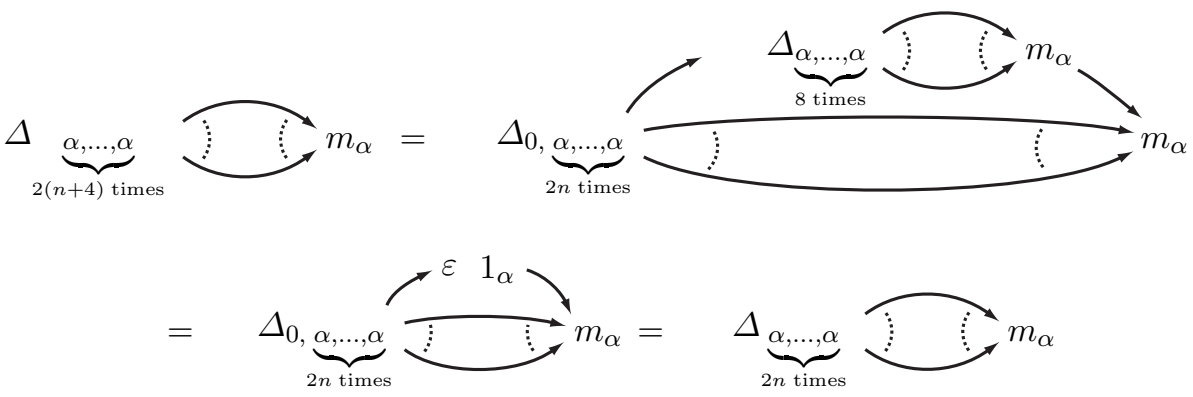

Fig. 15

Therefore $K_{H}\left(\xi_{n+4}^{k}\right)=K_{H}\left(\xi_{n}^{k}\right)$ for any $n \geq 1$. Hence, by computing by hand the values of $K_{H}\left(\xi_{n}^{k}\right)$ for $1 \leq n \leq 4$ and $0 \leq k \leq 1$, we obtain

$$
K_{H}\left(\xi_{n}^{0}\right)=4 \quad \text { and } \quad K_{H}\left(\xi_{n}^{1}\right)= \begin{cases}2 & \text { if } n \equiv 1(\bmod 2), \\ 4 & \text { if } n \equiv 0(\bmod 4), \\ 0 & \text { if } n \equiv 3(\bmod 4) .\end{cases}
$$

4.2. Example 2. We suppose that $\mathbb{k}$ is the field $\mathbb{C}$ of complex numbers. Let $\pi$ and $G$ be two finite groups, and $\phi: G \rightarrow \pi$ be a group homomorphism. Then $\phi$ induces a Hopf algebras morphism $F(\pi) \rightarrow F(G)$, given by $f \mapsto f \circ \phi$, whose image is central. Here $F(G)=\mathbb{C}^{G}$ and $F(\pi)=$ $\mathbb{C}^{\pi}$ denote the Hopf algebras of complex-valued functions on $G$ and $\pi$ respectively. By Section 1.2, this data leads to a Hopf $\pi$-coalgebra $H^{\phi}=$ $\left\{H_{\alpha}^{\phi}\right\}_{\alpha \in \pi}$. 
Let us describe this Hopf $\pi$-coalgebra more precisely. Denote by $\left(e_{g}\right)_{g \in G}$ the standard basis of $F(G)$ given by $e_{g}(h)=\delta_{g, h}$. Then, for any $\alpha, \beta \in \pi$, we have:

$$
\begin{aligned}
& H_{\alpha}^{\phi}=\sum_{g \in \phi^{-1}(\alpha)} \mathbb{C} e_{g}, \quad m_{\alpha}\left(e_{g} \otimes e_{h}\right)=\delta_{g, h} e_{g} \quad \text { for any } g, h \in \phi^{-1}(\alpha), \\
& 1_{\alpha}=\sum_{g \in \phi^{-1}(\alpha)} e_{g}, \quad \varepsilon\left(e_{g}\right)=\delta_{g, 1} \quad \text { for any } g \in \phi^{-1}(1), \\
& \Delta_{\alpha, \beta}\left(e_{g}\right)=\sum_{\substack{h \in \phi^{-1}(\alpha) \\
k \in \phi^{-1}(\beta) \\
h k=g}} e_{h} \otimes e_{k} \quad \text { for any } g \in \phi^{-1}(\alpha \beta), \\
& S_{\alpha}\left(e_{g}\right)=e_{g^{-1}} \quad \text { for any } g \in \phi^{-1}(\alpha) .
\end{aligned}
$$

Note that the Hopf $\pi$-coalgebra $H^{\phi}=\left\{H_{\alpha}^{\phi}\right\}_{\alpha \in \pi}$ is involutory and of finite type. Since $\operatorname{dim} H_{1}^{\phi}=\# \phi^{-1}\left(1_{G}\right)=\# \operatorname{ker} \phi$ is non-zero in the field $\mathbb{C}$, the invariant $K_{H^{\phi}}$ of pointed flat $\pi$-bundles over 3 -manifolds is well defined.

Proposition 10. Let $(\xi, \widetilde{x})$ be a pointed flat $\pi$-bundle over a 3-manifold $M$. Then

$$
K_{H^{\phi}}(\xi, \widetilde{x})=\#\left\{g: \pi_{1}(M, x) \rightarrow G \mid \phi \circ g=f\right\},
$$

where $x$ is the image of $\widetilde{x}$ under the bundle map of $\xi$ and $f: \pi_{1}(M, x) \rightarrow \pi$ is the monodromy of $\xi$ at $\widetilde{x}$.

Proof. Using the above explicit description of $H^{\phi}$, one easily sees that, for any $\alpha \in \pi$ and $g_{1}, \ldots, g_{m} \in \phi^{-1}(\alpha)$,

$$
m_{\alpha}= \begin{cases}1 & \text { if } g_{1}=g_{2}=\cdots=g_{m}, \\ 0 & \text { otherwise }\end{cases}
$$

and for any $\alpha_{1}, \ldots, \alpha_{n} \in \pi$ such that $\alpha_{1} \cdots \alpha_{n}=1$ and $g_{1} \in \phi^{-1}\left(\alpha_{1}\right), \ldots$, $g_{n} \in \phi^{-1}\left(\alpha_{n}\right)$,

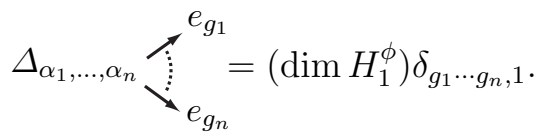

Let $(\xi, \widetilde{x})$ be a pointed flat $\pi$-bundle over a 3 -manifold $M$. Let $D=$ $(S, u, l)$ be a Heegaard diagram of genus $g$ of $M$. Orient it and color it by the monodromy $f$ of $\xi$ at $\widetilde{x}$. Denote by $\alpha=\left(\alpha_{1}, \ldots, \alpha_{g}\right)$ the color of $D$. Let $w_{1}, \ldots, w_{g}$ be the words in the alphabet $\left\{X_{1}, \ldots, X_{g}\right\}$ as in Section 2.1. For any $1 \leq i \leq g$, write $w_{i}\left(X_{1}, \ldots, X_{g}\right)=X_{k_{i, 1}}^{\varepsilon_{i, 1}} \cdots X_{k_{i, n_{i}}}^{\varepsilon_{i, i_{i}}}$ where $\varepsilon_{i, j}= \pm 1$ and $1 \leq k_{i, j} \leq g$. The non-zero factors of the tensor associated to a circle $u_{i}$ are 
of the form

$$
{ }_{e_{g_{i}}}^{e_{g_{i}}} m_{\alpha_{i}}=1 \quad \text { where } g_{i} \in \phi^{-1}\left(\alpha_{i}\right) \text {. }
$$

The non-zero factors of the tensor associated to a circle $l_{i}$ are of the form

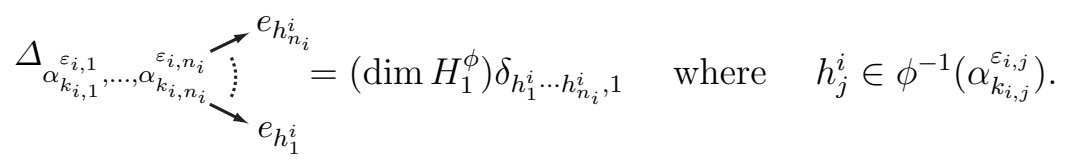

Now, at each crossing, that is, for $1 \leq p \leq g$ and $1 \leq q \leq n_{p}$, the contraction rule amounts to the equality of $h_{q}^{p}$ with $g_{k_{p, q}}^{\varepsilon_{p, q}}$. Therefore we get

$$
\begin{aligned}
& K_{H^{\phi}}(\xi, \widetilde{x}) \\
& =\left(\operatorname{dim} H_{1}^{\phi}\right)^{-g} \sum_{\substack{1 \leq i \leq g \\
1 \leq j \leq n_{i}}} \sum_{\substack{g_{i} \in \phi^{-1}\left(\alpha_{i}\right) \\
h_{j}^{i} \in \phi^{-1}\left(\alpha_{k_{i, j}}^{\varepsilon_{i, j}}\right)}}\left(\prod_{1 \leq p \leq g}\left(\operatorname{dim} H_{1}^{\phi}\right) \delta_{h_{1}^{p} \ldots h_{n_{p}}^{p}, 1}\right)\left(\prod_{\substack{1 \leq p \leq g \\
1 \leq q \leq n_{p}}} \delta_{h_{q}^{p}, g_{k_{p}, q}^{\varepsilon_{p}, q}}\right) \\
& =\sum_{1 \leq i \leq g} \sum_{g_{i} \in \phi^{-1}\left(\alpha_{i}\right)}\left(\prod_{1 \leq p \leq g} \delta_{w_{p}\left(g_{1}, \ldots, g_{g}\right), 1}\right) \\
& =\#\left\{\left(g_{1}, \ldots, g_{g}\right) \in G^{g} \mid w_{i}\left(g_{1}, \ldots, g_{g}\right)=1 \text { and } \phi\left(g_{i}\right)=\alpha_{i}, \forall 1 \leq i \leq g\right\} \\
& =\#\left\{g: \pi_{1}(M, x) \rightarrow G \mid \phi \circ g=f\right\} .
\end{aligned}
$$

\section{References}

[1] M. Atiyah, Topological quantum field theories, Publ. Math. IHES 68 (1989), 175-186.

[2] B. Enriquez, private communication, 2001.

[3] W. Fulton, Algebraic Topology, Springer, New York, 1995.

[4] G. Kuperberg, Involutory Hopf algebras and 3-manifold invariants, Internat. J. Math. 2 (1991), 41-66.

[5] J. Singer, Three-dimensional manifolds and their Heegaard diagrams, Trans. Amer. Math. Soc. 35 (1933), 88-111.

[6] M. E. Sweedler, Hopf Algebras, Benjamin, New York, 1969.

[7] - Integrals for Hopf algebras, Ann. of Math. (2) 89 (1969), 323-335.

[8] V. Turaev, Homotopy field theory in dimension 2 and group-algebras, preprint QA/9910010, 1999.

[9] - Homotopy field theory in dimension 3 and crossed group-categories, preprint GT/0005291, 2000.

[10] L. Vainerman, private communication, 2001.

[11] A. Virelizier, Algèbres de Hopf graduées et fibrés plats sur les 3-variétés, Ph.D. thesis, 2001.

[12] —, Hopf group-coalgebras, J. Pure Appl. Algebra 171 (2002), 75-122. 
[13] A. Virelizier, Graded quantum groups and quasitriangular Hopf algebras, Comm. Algebra 33 (2005), 3029-3050.

Department of Mathematics

University of California

Berkeley, CA 94720-3840, U.S.A.

E-mail: virelizi@math.berkeley.edu

Received 14 February 2005;

in revised form 22 July 2005 